\title{
Flora of community managed forests of Palpa district, western Nepal
}

\author{
Pratiksha Shrestha ${ }^{1} \bowtie$, Ram Prasad Chaudhary ${ }^{2}$, \\ Krishna Kumar Shrestha', Dharma Raj Dangol ${ }^{3}$
}

\author{
${ }^{1}$ Central Department of Botany, Tribhuvan University, Kathmandu, Nepal \\ ${ }^{2}$ Research Center for Applied Science and Technology (RECAST), Kathmandu, Nepal \\ ${ }^{3}$ Natural History Museum, Tribhuvan University, Swayambhu, Kathmandu, Nepal
}

\begin{abstract}
Floristic diversity is studied based on gender in two different management committee community forests (Barangdi-Kohal jointly managed community forest and Bansa-Gopal women managed community forest) of Palpa district, west Nepal. Square plot of $10 \mathrm{~m} \times 10 \mathrm{~m}$ size quadrat were laid for covering all forest areas and maintained minimum $40 \mathrm{~m}$ distance between two quadrats. Altogether 68 plots (34 in each forest) were sampled. Both community forests had nearly same altitudinal range, aspect and slope but differed in different environmental variables and members of management committees. All the species present in quadrate and as well as outside the quadrate were recorded for analysis. There were 213 species of flowering plant belonging to 67 families and 182 genera. Barangdi-Kohal JM community forest had high species richness i.e. 176 species belonging to 64 families and 150 genera as compared to Bansa-Gopal WM community forest with 143 species belonging to 56 families and 129 genera. According to different life forms and family and genus wise jointly managed forest have high species richness than in women managed forest. Both community forests are banned for fodder, fuel wood and timber collection without permission of management comities. There is restriction of grazing in JM forest, whereas no restriction of grazing in WM forest.
\end{abstract}

Key words: management practice, jointly managed forest, women managed forest

\section{INTRODUCTION}

Nepal shares $0.1 \%$ of the total land area of the world but harbors over $3 \%$ of the world's flowering plants. The number of flowering plant enumerated in Nepal is 6,973 species of angiosperm and 26 species of gymnosperm (MoFSC, 2014). Nepal ranks 10th position in Asia and 27th in world for richness in flowering plant diversity (BPP, 1995). Flora refers to the brief taxonomic treatment of all plants occurring in a particular geographical location which generates a comprehensive account. A complete flora of a country is necessary to reflect the whole plant diversity of that country (Shakya et al., 1997).sCommunity forestry program especially focuses on protection and production timber yielding species rather than lower herbs and shrubs in community forests. Non-timber and low quality yielding species are indiscriminately removed during various management practices (Belbase, 1999; Shrestha, 2005). The role of women in sustainable resource management and women's indigenous 
knowledge are greater than that of men with respect to species and use. So the participation of women in community forest is increasing day by day (Upadhyay, 2005; Agarwal, 2009).

The pattern of species richness with respect to different management committees like women managed community forest and jointly managed community forest has not been studied for generating scientific data. Only the condition of forest on the basis of participation in management committee and decision making were analysed by different persons (Bhattarai, 1997; Agarwal \& Ostrom, 2001; Upadhaya, 2005; Agarawal, 2009; Gurung, 2013). In Palpa district, there are some works done related to species richness and floristic diversity in different area (Shrestha et al., 2003; Bhandari, 2006; Mahato, 2006; Ghubhaju, 2009). However, no any work done previously by researchers in this study area. This paper focus to generate scientific data on species diversity in two community forests having different management committees based on gender.

\section{MATERIALS AND METHODS}

\section{Study area}

The study was conducted in Palpa district, located in Lumbini zone in the western development region of Nepal. It is located between $27^{\circ} 34^{\prime}-27^{\circ} 57^{\prime} \mathrm{N}$ and $83^{\circ} 15^{\prime}-83^{\circ} 22^{\prime} \mathrm{E}$ in the central part of Nepal. Palpa is a hilly district bounded by Nawalparasi and Tanahun district towards east, Arghakhachi and Gulmi district towards west, Syanja and Tanahun district towards north and Nawalparasi and Rupandehi district towards south. The total land area of this district is about $1,373 \mathrm{sq}$. $\mathrm{km}$. with approximate length of $70 \mathrm{~km}$ and breadth of $20 \mathrm{~km}$. The altitude varies from tropical (about $213 \mathrm{~m})$ to upper subtropical region $(1,900 \mathrm{~m})$. About $711 \mathrm{sq}$. $\mathrm{km}$ area $(51.8 \%)$ of Palpa district is covered by forests. Among total forest cover, $18 \%$ lies in Churiya range and $82 \%$ lies in Mahabharat range. The Palpa district comprises of 634 community forest cover an area of $321 \mathrm{sq}$. km (DFO, 2015). This study is mainly comprised of the tropical zone and Schima- Castanopsis forest type. Both community forests are community forests and there is only 4 year's difference in development of community forest. However, the physiographic and floristic such as altitude, forest type, slope, aspects are same between the forests.

The study is mainly focused on assessment of floristic diversity in two community forests: one is Barangdi-Kohal of Madanpokhara VDC which is jointly managed (JM) community forest handed over to community in 2051 B.S. (1994) where the management committee has both male and female members to regulate the forest utilization and another is Bansa-Gopal women managed (WM) community forest of Pravas, Tansen municipality, handed over to community in 2055 B.S. (1998) where all the members of forest management committee and users groups who manage the forest are women.

For floristic diversity study, four field visits were made in between April 2013 to July 2014 . The first field visit was from 12th to 16th April 2013 for the selection of site and collection of general information about forests from the local informants as well as management committees. The second field visit was done from June 25th to 2nd July 2013 for and plant specimens were collected. Third and fourth visit was conducted in 16th to 20th October 2013 and February 4th to 7th, 2014 respectively for collection of plant specimens which were not collected in previous field visit. 
Most of the plant species was identified in the field with the help of standard floras (Malla et al., 1984; Polunin \& Staintion 1984; Stainton, 1988; Shrestha 1998). Then unidetified plants were identified later with the help of experts. All the herbarium specimens were tallied and identified later at TUCH, Kirtipur and KATH Godawari, Lalitpur. Only the angiosperms and gymnosperms were collected from the study area. The species which were not in flowering/ fruiting stage during field visit and most popular species as voucher specimens were not collected. Digital photographs were taken of most of the plants for future references. The collected specimens were tagged, pressed and dried naturally and prepare herbarium by following technique of Bridson \& Forman (1989). Generally 2 to 3 specimens for each species were collected. Nomenclature of plant species follows Press et al. (2000). The specimens were deposited in TUCH, KATH and TMC Tansen, Palpa.

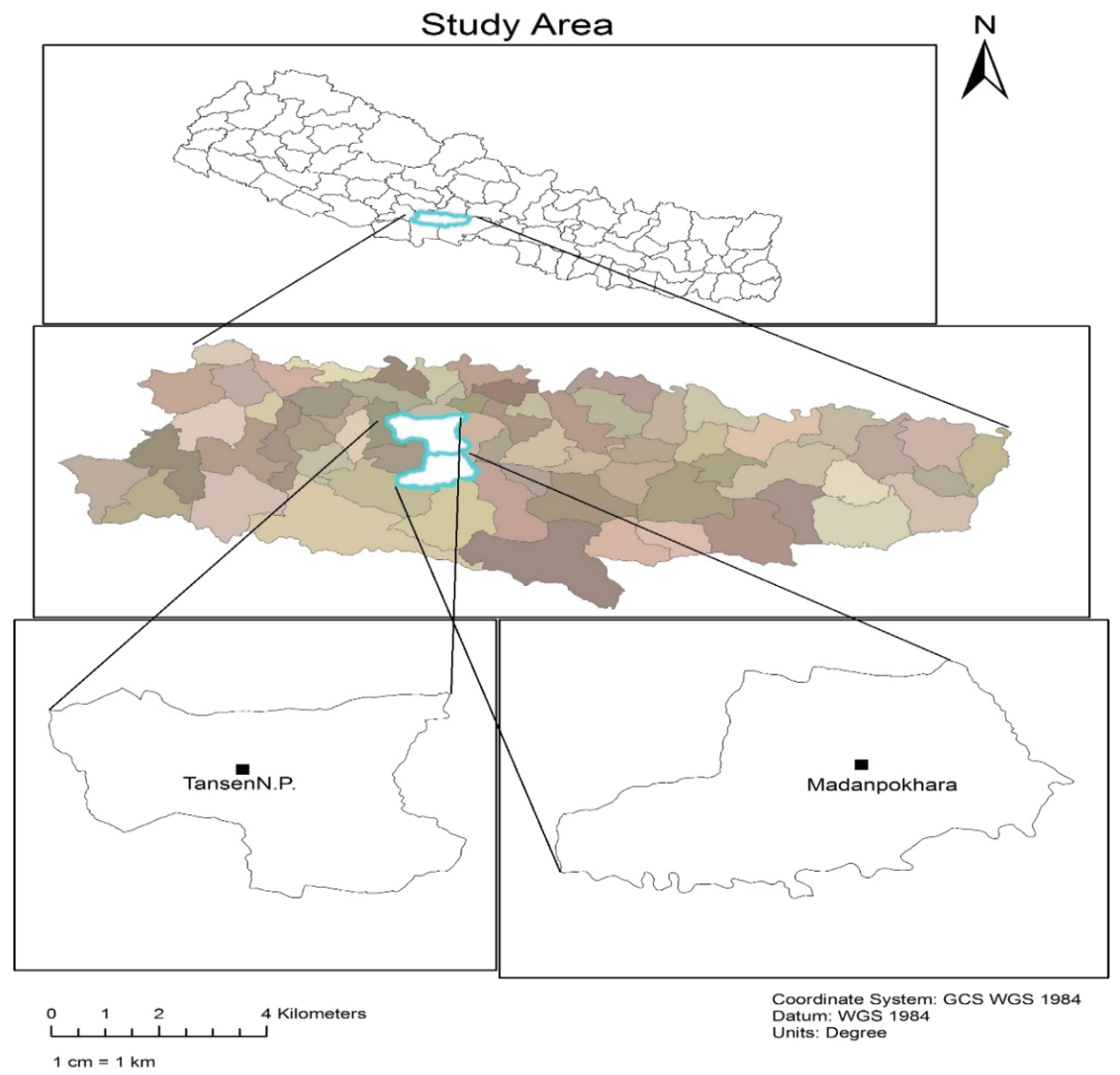

FIG. 1. Map of the study area. 


\section{RESULTS AND DISCUSSION}

\section{Floristic diversity}

Altogether, 213 species of flowering plants belonging to 64 families and 182 genera (excluding 3 unidentified species), which included 46 trees, 36 shrubs, 107 herbs and 24 climbers were recorded in two community forests of Palpa district (appendix 1). Out of 213 species, 178 species belong to dicotyledons, 34 species of monocotyledons and 1 species of gymnosperm. The largest family was Leguminosae which consist 15 genera and 24 species. The second largest genera were Compositae comprising 20 genera and 21 species.

In Barangdi-Kohal jointly managed community forest, 176 species belonging to 64 families and 150 genera were recorded. Among them, 148 species belong to dicotyledons, 27 species to monocotyledons and 1 species to gymnosperm. Out of 176 species, 78 species belong to herbs, 31 species to shrubs, 45 species to trees and 22 species to climbers. Fabaceae was found to be largest family comprising 14 genera and 20 species. The other larger families were Poaceae (13 genera and 13 species), Asteraceae (11 genera and 12 species), Lamiaceae (6 genera and 10 species), Euphorbiaceae ( 7 genera and 9 species) and Moraceae (3 genera and 8 species).

In Bansa-Gopal women managed community forest, 143 species belonging to 56 families and 129 genera were recorded. Among them 118 species belong to dicots, 24 species to monocots and 1 species to gymnosperm (table 1). Out of 143 species, 73 species belong to herbs, 18 species to shrubs, 37 species to trees and 15 species to climbers.

In jointly managed forest, high number of families and genera were found as compared to women managed forest. Also according to different life forms higher number of species were found in jointly managed forest than in women managed forest.

TABLE 1. The number of family, genus, and species of Dicotyledos, Monocotyledons and Gymnosperms in two community forests of Palpa district, Nepal.

\begin{tabular}{|l|c|c|c|c|c|c|}
\hline \multirow{2}{*}{ Major taxa } & \multicolumn{3}{|c|}{ JM forest } & \multicolumn{3}{c|}{ WM forest } \\
\cline { 2 - 7 } & Family & Genus & Species & Family & Genus & Species \\
\hline Dicot & 52 & 122 & 148 & 48 & 106 & 118 \\
\hline Monocot & 11 & 27 & 27 & 7 & 22 & 24 \\
\hline Gymnosperm & 1 & 1 & 1 & 1 & 1 & 1 \\
\hline Total & 64 & 150 & 176 & 56 & 129 & 143 \\
\hline
\end{tabular}

Of the 64 families, the Leguminosae, Compositae, Poaceae, and Lamiaceae were four largest families with more than 10 species (fig. 2). Leguminosae and Lamiaceae were two dominant families in JM forest whereas Compositae and Poaceae were two dominant families in WM forest. The family Poaceae shared highest common species (9) in JM and WM. JM forest had highest unique species (13) of Leguminosae and WM had 9 unique species of Compositae (fig. 2). 


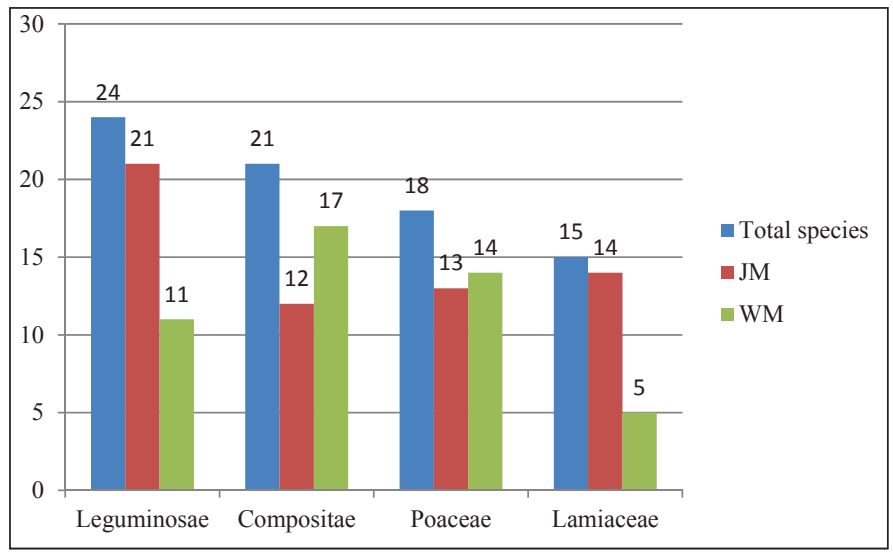

FIG. 2. Four major families with more than 10 species in two community forests of Palpa, Nepal.

\section{Life form diversity}

The number of climbers, herbs, shrubs and trees are highest in JM forests in compared to WM forests (table 2).

TABLE 2. Life forms of plants of two community forests, Palpa district, Nepal.

\begin{tabular}{|c|c|c|c|c|c|}
\hline Forests & Climbers & Herbs & Shrubs & Trees & Total species \\
\hline JM Forest & 22 & 78 & 31 & 45 & 176 \\
\hline WM forest & 15 & 73 & 18 & 37 & 143 \\
\hline
\end{tabular}

In JM forest, 9 species of climbers, 34 species of herbs, 18 species of shrubs and 9 species of trees are found only in this forest (Table 3). Whereas, 2 species of climbers, 29 species of herbs, 12 species of shrubs and 1 species of trees are found only in women managed forest. 13 species of climbers, 44 species of herbs, 13 species of shrubs and 36 species of trees are found in both community forests (table3).

TABLE 3. Unique and common of plant species (by life forms) in two community forests.

\begin{tabular}{|l|c|c|c|c|}
\hline Life forms & JM only (\%) & WM only (\%) & JM+WM (\%) & Total (\%) \\
\hline Climbers & $9(37.50)$ & $2(8.33)$ & $13(54.17)$ & $24(100.00)$ \\
\hline Herbs & $34(31.78)$ & $29(27.10)$ & $44(41.12)$ & $107(100.00)$ \\
\hline Shrubs & $18(37.50)$ & $5(37.50)$ & $13(36.11)$ & $36(100.00)$ \\
\hline Trees & $9(19.56)$ & $1(2.17)$ & $36(78.26)$ & $46(100.00)$ \\
\hline Total & 70 & 37 & 106 & $\mathbf{2 1 3}(100.00)$ \\
\hline
\end{tabular}

The study areas were dominated by Schima wallichi and Castanopsis indica. In the study area, there were found many medicinal and highly valuable plant which are conserved by nationally and international organization. 


\section{Status of flora}

\begin{tabular}{|l|l|l|}
\hline \multicolumn{1}{|c|}{ Plant species } & \multicolumn{1}{c|}{ Forests } & \multicolumn{1}{|c|}{ Status } \\
\hline Asparagus racemosus & JM forest & Nationally threatened, Vulnerable \\
\hline Curculigo orchioides & JM forest & Nationally threatened, Vulnerable \\
\hline Piper longum & JM forest & Nationally threatened, Vulnerable \\
\hline Rauvolfia serpentina & JM forest & $\begin{array}{l}\text { Nationally threatened, Vulnerable, Plant species } \\
\text { protected under Forest Act 1993; IUCN threat category } \\
\text { (Endangered) }\end{array}$ \\
\hline Bombax ceiba, & JM & Plant species protected under Forest Act 1993 \\
\hline Dioscorea deltoidea & JM \& WM & CITES LIST (appendix II) \\
\hline
\end{tabular}

Jointly managed forest is rich in many threatened and protected plants species by government of Nepal as compared to women managed forest. Only Dioscorea deltoidea are found in women managed forest which falls under CITES Appendix II, but other species are not found in this forest.

The floral diversity of jointly managed community forest is higher than the women managed forest. It may be due to collection pattern for feeding animals and for fuelwood. In WM forests, women collect fodder and fuel wood species and mainly focus to conserve tree species but the jointly managed community forest focus to conserve the all species. Both community forests are banned for fodder, fuel wood and timber collection without permission of management committees. There is restriction for grazing in jointly managed forest, whereas no restriction of grazing in women managed forest which also affect the availability of plant species.

\section{ACKNOWLEDGEMENTS}

The authors are thankful to the Central Department of Botany, Tribhuvan University, Kirtipur for giving permission to conduct this work. The authors are grateful to peoples of two community forests and also grateful to Cornell Nepal Study Program (CNSP) and Tansen Guthi Kathmandu for providing partial financial support.

\section{REFERENCES}

Agrawal, A; Ostrom, E (2001) Collective action, property rights and decentralization in resource use in India and Nepal. Political Society 29: 485-514.

Agrawal, B (2009) Gender and forest conservation: the impact of women's participation in community forest governance. Ecological Economics 68: 2785-2799.

Belbase, N (1999) National implementation of the convention on biological diversity, policy and legislative requirement. IUCN, Kathmandu, Nepal.

Bhandari, R K (2006) Floristic study of Dovan village development committee, Palpa district. M. Sc. Dissertation, Central Department of Botany, Tribhuvan University, Kirtipur, Kathmandu, Nepal.

Bhattarai, N K (1997) Role of Women in the use and conservation of biodiversity; studies in the Nepal Himalaya. Banko Jankari 7: 3-8. 
BPP (1995) Biodiversity profile of the Terai/Siwalik physiographic zones. Biodiversity Profile Project Publication Number 12. Department of National Park and Wildlife Conservation, Kathmandu Nepal.

Bridson, D; Forman, L (1989) The Herbarium handbook. Royal Botanic Garden. Kew, UK (3rd edition). haudhary, R P (1998) Biodiversity in Nepal: status and conservation. Tecpress, Bangkok, Thailand.

Glowka, L; Burhenne-Guilmin, F; Sygne, H (1994) A guide to the convention on biological diversity. IUCN Gland, Switzerland.

Gubhaju, M R (2009) Non-Timber Forest Products (NTFPs) in community forests of Dovan, Palpa: diversity, population status and patterns of utilization. M. Sc. Dissertation, Central Department of Botany, Tribhuvan University, Kirtipur, Kathmandu, Nepal.

Gurung, P (2013) Role of participation of women in community forest management: a case study of dharapani women community forest, Bharatpokhari VDC, Kaski, Nepal. M. Sc. Dissertation, Prithivi Narayan Campus, Pokhara, Nepal.

Mahato, R B (2006) Diversity, use and coservation of plants in Palpa district, Nepal. PhD. Thesis, Central Department of Botany, Tribhuvan University, Kirtipur, Kathmandu, Nepal.

Malla, S B; Rajbhandari, S B; Shrestha, T B; Adhikari, P M; Shakya, P R (eds) (1986). Flora of Kathmandu valley. Government of Nepal. Ministry of Forests and Soil Conservation, Kathmandu, Nepal.

Maren, I E ; Bhattarai, K R ; Chaudhary, R P (2013) Forest ecosystem services and biodiversity in contrasting Himalayan forest management systems. Environmental Conservation 41(1): 73-83.

MoFSC (2014) Nepal biodiversity strategy and action plan 2014-2020. Government of Nepal, Ministry of Forests and Soil Conservation, Kathmandu, Nepal.

Polunin, O; Stainton, J D A (1984) Flowers of the Himalaya. Oxford University Press, New Delhi, India.

Press, J R; Shrestha, K K; Sutton, D A (2000) Annotated checklist of flowering plants of Nepal. Natural History Museum, London, UK and Central Department of Botany, Tribhuvan University. Kathmandu, Nepal.

Shakya, P R; Adhikari, M K; Rajbhandari, K R; Chaudhary, R P; Shrestha, K K (1997) Flora of Nepal. Presented at International Seminar-Cum-Workshop on Flora of Nepal, Kathmandu, Nepal.

Shrestha, K K; Tiwari, N N; Rajbhandary, S; Shrestha, S; Upreti, Y; Poudel, R C (2003) Non-timber forest products (NTFPs) in the critical bottlenecks and corridors of Terai Arc Landscape-Nepal: Documentation, utilization, trade and people livelihood. A Project Report Submitted to WWF Nepal programme by Ethnobotanical Society of Nepal (ESON), Kathmandu, Nepal.

Shrestha, B B (2005) Fuel wood consumption, management and regeneration of two community forest in central Nepal. Himalayan Journal of Science 3(5): 75-80.

Shrestha, K (1998) Dictionary of Nepalese plant names. Mandala Book Point, Kathmandu, Nepal.

Shrestha, T B; Joshi, R M (1996) Rare, endemic and endangered plants of Nepal. WWF Nepal Program, Kathmandu, Nepal.

Stainton, J D A (1972) Forest of Nepal. The Camelot Press Ltd., London and Southampton, UK.

Staiton, A (1988) Flowers of the Himalaya: a supplement, Oxford University Press, India.

Upadhyay, B (2005) Women and natural resource management. Illustrations from India and Nepal. Natural Resource FORUM 29(3): 224-232. 
Shrestha et al.: Flora of community managed forests

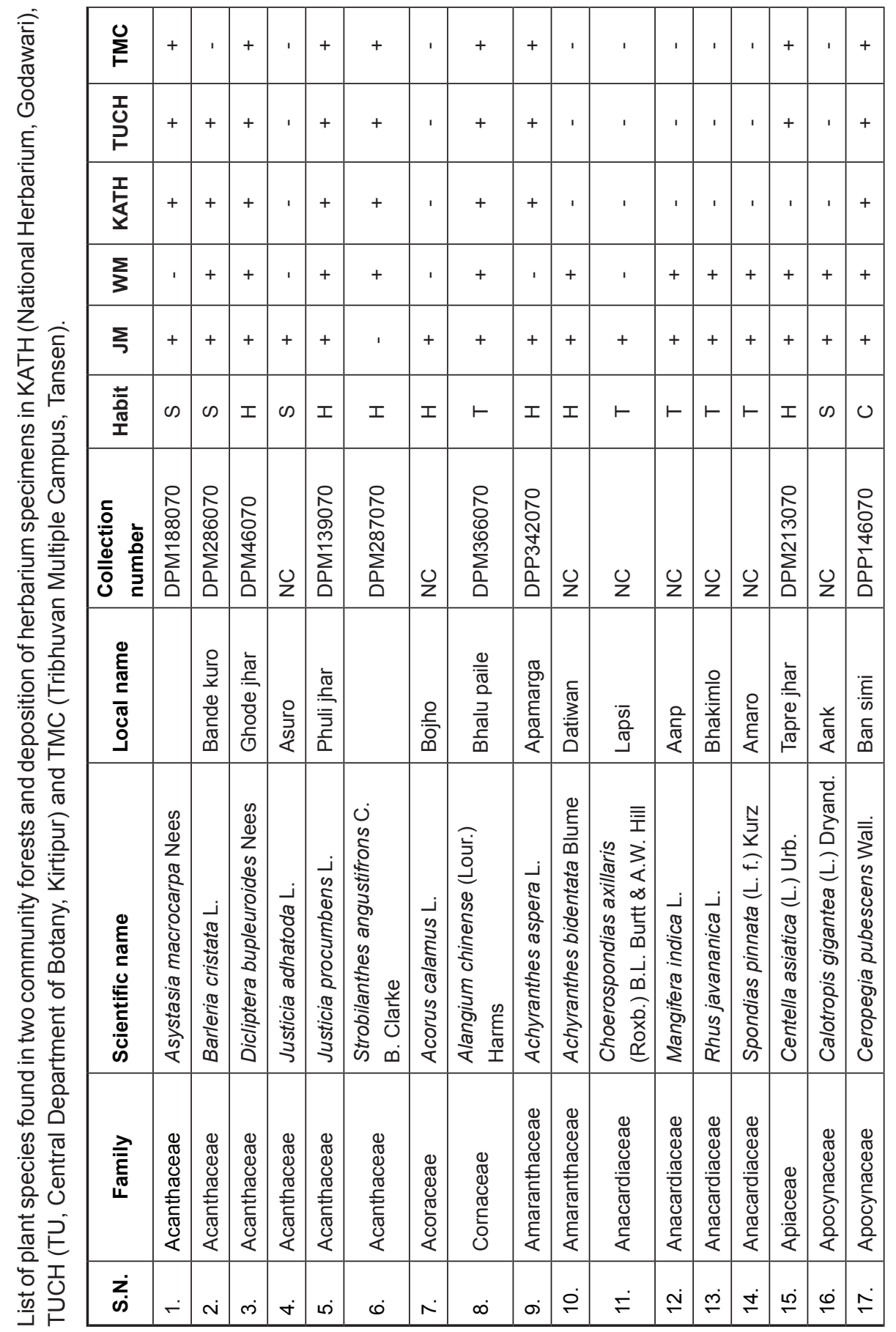




\begin{tabular}{|c|c|c|c|c|c|c|c|c|c|c|c|c|c|c|c|c|c|}
\hline . & + & ' & 1 & + & ' & ' & 1 & + & ' & ' & ' & + & I & + & ' & + & + \\
\hline ' & + & . & 1 & + & ' & ' & , & + & + & I & ' & + & I & + & + & + & + \\
\hline ' & + & ' & + & + & 1 & 1 & 1 & + & + & . & ' & + & + & + & + & + & + \\
\hline+ & + & + & ' & + & + & ' & 1 & + & + & + & + & + & ' & + & + & I & + \\
\hline ' & + & ' & + & + & + & + & + & ' & ' & + & + & + & + & + & + & + & I \\
\hline 0 & 0 & $\vdash$ & I & $I$ & I & $I$ & $I$ & I & I & I & I & I & I & I & I & I & I \\
\hline U & $\begin{array}{l}\text { R } \\
\text { o } \\
\bar{m} \\
\sum_{0}^{m} \\
0\end{array}$ & U & $\begin{array}{l}\text { P } \\
\text { O } \\
\stackrel{5}{+} \\
\sum_{0}^{N} \\
0\end{array}$ & $\begin{array}{l}0 \\
0 \\
8 \\
0 \\
0 \\
0 \\
0\end{array}$ & z & & $\mathbf{z}$ & 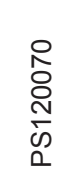 & 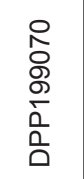 & U & u & $\begin{array}{l}0 \\
o \\
o \\
\dot{J} \\
\sum_{0} \\
0\end{array}$ & $\begin{array}{l}0 \\
0 \\
0 \\
0 \\
\sum_{0}^{m} \\
0 \\
0\end{array}$ & $\begin{array}{l}\text { R } \\
8 \\
\frac{0}{0} \\
0 \\
0 \\
0\end{array}$ & $\begin{array}{l}0 \\
0 \\
o \\
0 \\
\stackrel{1}{N} \\
\sum_{0} \\
0\end{array}$ & 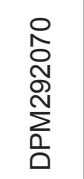 & 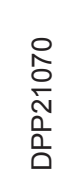 \\
\hline & $\begin{array}{l}\frac{\pi}{\sqrt{0}} \\
\frac{C}{\sigma} \\
\frac{\pi}{0} \\
\frac{1}{0} \\
\frac{0}{2} \\
0\end{array}$ & 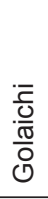 & 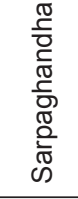 & 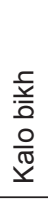 & 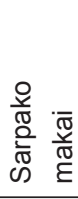 & 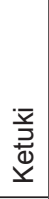 & $\begin{array}{l}\frac{0}{\frac{T}{2}} \\
\frac{2}{2}\end{array}$ & & & 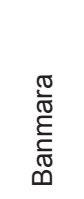 & 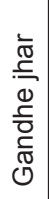 & 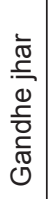 & 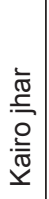 & $\begin{array}{l}\overline{\mathbb{D}} \\
\frac{Q}{Q} \\
\stackrel{\vec{D}}{F}\end{array}$ & $\begin{array}{l}\stackrel{0}{\xi} \\
\underline{y}\end{array}$ & 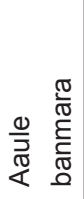 & 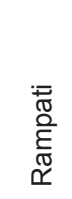 \\
\hline 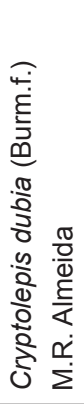 & 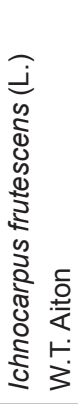 & 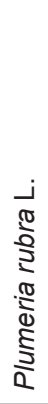 & 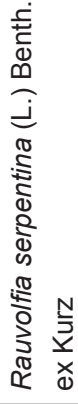 & 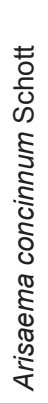 & 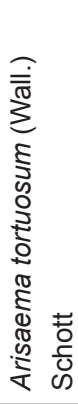 & 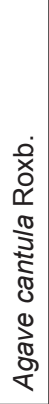 & 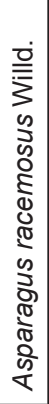 & 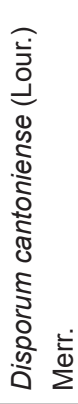 & 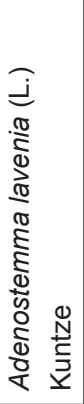 & 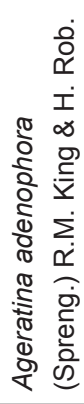 & 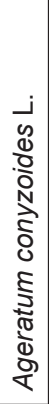 & 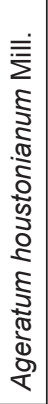 & 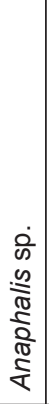 & 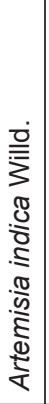 & $\begin{array}{l}\dot{1} \\
\mathbb{0} \\
0 \\
0 \\
\frac{0}{2} \\
0 \\
0 \\
\frac{0}{0} \\
00 \\
00\end{array}$ & 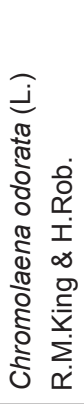 & 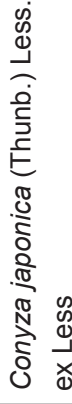 \\
\hline 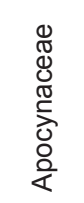 & 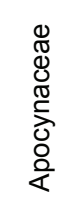 & 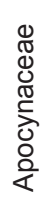 & 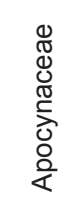 & $\begin{array}{l}\mathbb{\Xi} \\
\mathbb{8} \\
0 \\
\mathbb{0} \\
\frac{\pi}{<}\end{array}$ & $\begin{array}{l}0 \\
\mathbb{\pi} \\
\mathbb{0} \\
\mathbb{0} \\
\frac{\pi}{4}\end{array}$ & 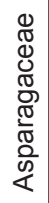 & $\begin{array}{l}\mathbb{0} \\
\mathbb{N} \\
\mathbb{8} \\
0 \\
\mathbb{0} \\
\mathbb{\pi} \\
\frac{\pi}{\pi} \\
\frac{2}{0} \\
\frac{0}{4}\end{array}$ & 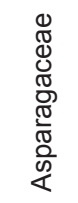 & $\begin{array}{l}\frac{\pi}{\pi} \\
\frac{\pi}{50} \\
0 \\
\frac{0}{E} \\
0 \\
0\end{array}$ & $\begin{array}{l}\frac{0}{\pi} \\
\frac{\pi}{0} \\
0 \\
\frac{0}{E} \\
0 \\
0\end{array}$ & $\begin{array}{l}\frac{0}{0} \\
\frac{\pi}{0} \\
0 \\
\frac{0}{\varepsilon} \\
\stackrel{0}{0} \\
0\end{array}$ & $\begin{array}{l}\frac{0}{0} \\
\frac{\pi}{5} \\
0 \\
\frac{0}{\varepsilon} \\
0 \\
0\end{array}$ & $\begin{array}{l}\frac{1}{0} \\
\frac{\pi}{50} \\
0 \\
\frac{0}{\varepsilon} \\
0 \\
0\end{array}$ & $\begin{array}{l}\frac{\pi}{\pi} \\
\frac{\pi}{5} \\
0 \\
\frac{0}{\varepsilon} \\
0 \\
0\end{array}$ & $\begin{array}{l}\frac{0}{0} \\
\frac{\pi}{00} \\
0 \\
\frac{0}{E} \\
0 \\
0\end{array}$ & 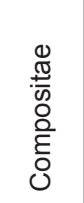 & $\begin{array}{l}\frac{0}{\frac{\pi}{5}} \\
\frac{0}{0} \\
0 \\
\frac{0}{\varepsilon} \\
0\end{array}$ \\
\hline$\stackrel{\infty}{-}$ & $\stackrel{\sigma}{-}$ & $\stackrel{N}{ }$ & $\bar{N}$ & న & $\stackrel{N}{N}$ & $\stackrel{\dot{N}}{ }$ & $\stackrel{2}{\sim}$ & 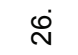 & $\hat{N}$ & $\stackrel{\infty}{N}$ & $\stackrel{\mathbf{N}}{ }$ & ळి & $\dot{m}$ & లిં & m & ले & లొ \\
\hline
\end{tabular}


Shrestha et al.: Flora of community managed forests

\begin{tabular}{|c|c|c|c|c|c|c|c|c|c|c|c|c|c|c|c|c|c|c|}
\hline+ & + & + & + & 1 & ' & + & + & I & + & I & + & ' & + & + & ' & ' & ' & ' \\
\hline+ & + & + & + & 1 & ' & + & + & 1 & + & + & + & ' & + & + & & . & ' & + \\
\hline+ & + & + & + & + & ' & + & + & + & + & + & + & ' & + & + & + & I & + & + \\
\hline+ & + & + & + & + & + & + & ' & 1 & + & + & + & ' & + & + & + & + & + & + \\
\hline ' & + & ' & ' & ' & + & I & + & + & I & + & ' & + & + & + & ' & + & + & + \\
\hline$I$ & $I$ & I & $I$ & $I$ & $I$ & $I$ & $\omega$ & $I$ & $I$ & I & $I$ & $\omega$ & $\vdash$ & $I$ & I & $\vdash$ & I & I \\
\hline 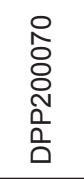 & 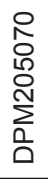 & 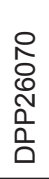 & 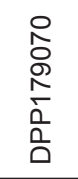 & 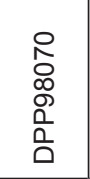 & $\mathbf{u}$ & $\begin{array}{l}0 \\
0 \\
0 \\
6 \\
\vdots \\
0 \\
0\end{array}$ & $\begin{array}{l}0 \\
0 \\
\circ \\
0 \\
\sum_{0} \\
0 \\
0\end{array}$ & $\begin{array}{l}\text { 응 } \\
8 \\
8 \\
\frac{0}{n} \\
\frac{0}{0}\end{array}$ & 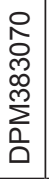 & $\begin{array}{l}0 \\
\stackrel{0}{\circ} \\
8 \\
\frac{0}{2} \\
\frac{0}{0} \\
0\end{array}$ & \begin{tabular}{l}
0 \\
0 \\
$⿱ 0$ \\
\multirow{O}{O}{} \\
$\sum_{0}$ \\
0 \\
0
\end{tabular} & $\mathcal{u}$ & 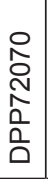 & 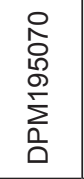 & 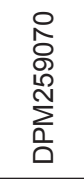 & $\mathcal{U}$ & 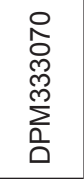 & 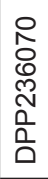 \\
\hline 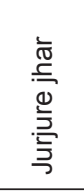 & 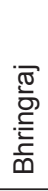 & 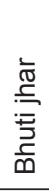 & $\begin{array}{l}\bar{\Xi} \\
\text { 은 } \\
\text { 임 }\end{array}$ & $\begin{array}{l}\frac{\pi}{\pi} \\
\stackrel{\frac{\pi}{\pi}}{\pi} \\
\frac{2}{\pi} \\
\frac{\pi}{\pi} \\
\omega\end{array}$ & 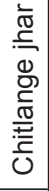 & 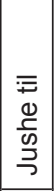 & 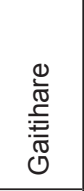 & & 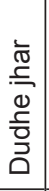 & $\begin{array}{l}\frac{1}{2} \\
\frac{2}{0} \\
0\end{array}$ & 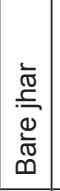 & $\begin{array}{l}\frac{0}{5} \\
\frac{5}{0}\end{array}$ & & 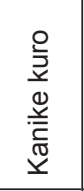 & 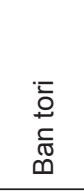 & $\begin{array}{l}\mathbb{0} \\
\frac{0}{\pi} \\
\frac{\pi}{0} \\
\frac{0}{\pi} \\
0\end{array}$ & $\begin{array}{l}\frac{0}{\pi} \\
\frac{0}{2} \\
\frac{10}{4}\end{array}$ & 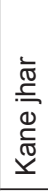 \\
\hline 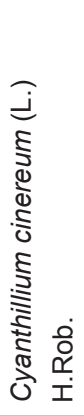 & 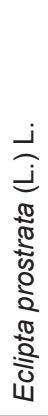 & 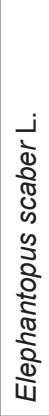 & 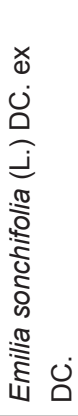 & 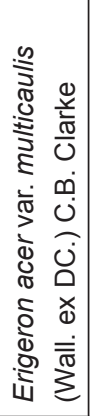 & 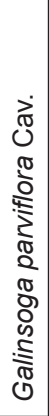 & 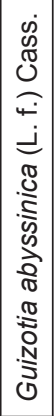 & 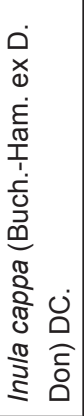 & 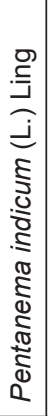 & 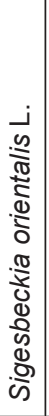 & 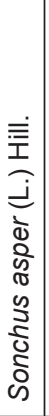 & 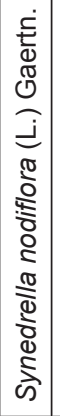 & 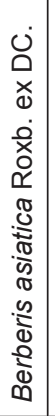 & 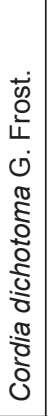 & 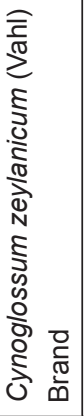 & 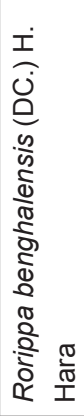 & 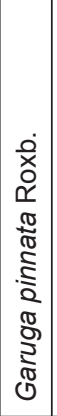 & 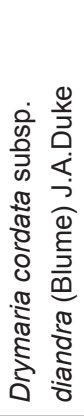 & 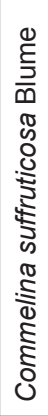 \\
\hline 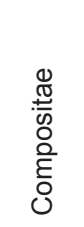 & $\begin{array}{l}\frac{\pi}{\pi} \\
\frac{\pi}{00} \\
0 \\
\frac{0}{E} \\
0 \\
0\end{array}$ & $\begin{array}{l}\frac{\pi}{\pi} \\
\frac{\pi}{50} \\
\frac{0}{0} \\
\text { हे } \\
0\end{array}$ & 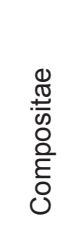 & 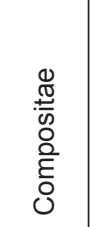 & 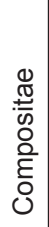 & 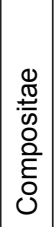 & 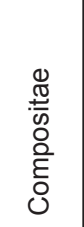 & 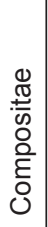 & 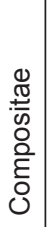 & $\begin{array}{l}\frac{\pi}{\pi} \\
\frac{\pi}{5} \\
0 \\
\frac{0}{\varepsilon} \\
0 \\
0\end{array}$ & 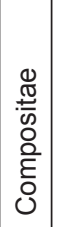 & 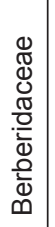 & 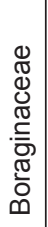 & 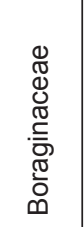 & 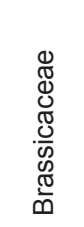 & 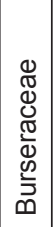 & 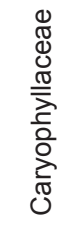 & 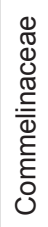 \\
\hline 户্ & $\hat{m}$ & $\stackrel{\infty}{\infty}$ & த் & \& & $\check{\nabla}$ & 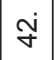 & 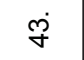 & $\forall$ & $\dot{q}$ & $\dot{\theta}$ & 守 & $\stackrel{\infty}{+}$ & 子 & ద & 5 & ธ่ & లి & ட் \\
\hline
\end{tabular}




\begin{tabular}{|c|c|c|c|c|c|c|c|c|c|c|c|c|c|c|c|c|c|}
\hline+ & ' & 1 & + & 1 & ' & + & ' & ' & + & + & ' & + & I & + & ' & I & ' \\
\hline+ & ' & ' & + & + & + & + & + & ' & + & + & + & + & I & + & I & I & ' \\
\hline+ & I & + & + & + & I & + & I & , & + & + & + & + & I & + & + & 1 & I \\
\hline ' & + & + & , & , & ' & + & + & ' & + & + & + & + & + & + & + & + & I \\
\hline+ & + & ' & + & + & + & + & + & + & + & + & I & + & + & + & I & + & + \\
\hline 0 & 0 & $I$ & 0 & 0 & 0 & 0 & 0 & 0 & $I$ & $I$ & I & 0 & $\vdash$ & $I$ & $I$ & $\vdash$ & $\vdash$ \\
\hline 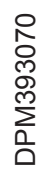 & z & $\begin{array}{l}\text { 웅 } \\
\text { o } \\
0 \\
0 \\
0 \\
0 \\
0 \\
0\end{array}$ & $\begin{array}{l}0 \\
0 \\
\text { o } \\
\text { 仓 } \\
\sum_{0} \\
0 \\
0\end{array}$ & 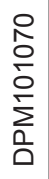 & $\begin{array}{l}0 \\
0 \\
\& \\
0 \\
\sum_{0}^{10} \\
0\end{array}$ & 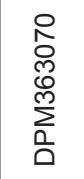 & 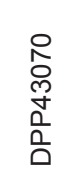 & U & 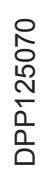 & 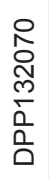 & 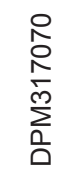 & 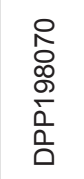 & U & 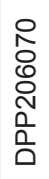 & $\begin{array}{l}0 \\
\circ \\
\circ \\
\stackrel{N}{ } \\
\frac{0}{0} \\
0\end{array}$ & $\mathbf{z}$ & u \\
\hline $\begin{array}{l}\text { 䇈 } \\
\text { 号 } \\
\text { 心 }\end{array}$ & 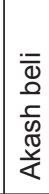 & & 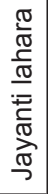 & & 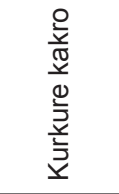 & 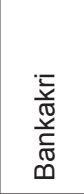 & 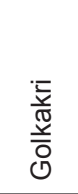 & 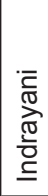 & 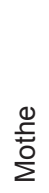 & $\begin{array}{l}\stackrel{0}{ \pm} \\
\stackrel{0}{0}\end{array}$ & & 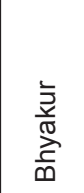 & 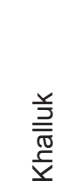 & 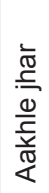 & 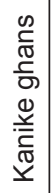 & 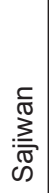 & 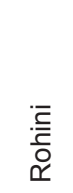 \\
\hline 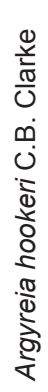 & 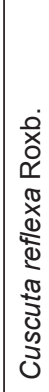 & 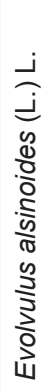 & 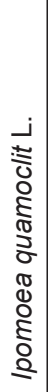 & $\begin{array}{l}0 \\
\text { D } \\
\mathbb{D} \\
\frac{\mathbb{d}}{0} \\
0 \\
0\end{array}$ & 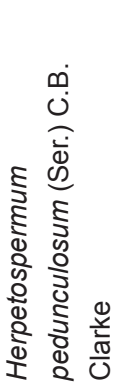 & 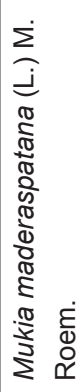 & 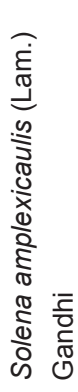 & 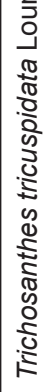 & 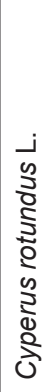 & 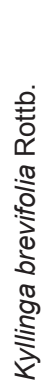 & 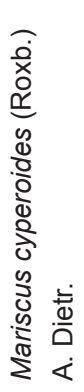 & 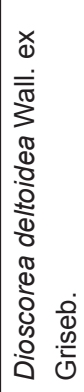 & 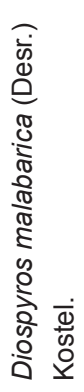 & 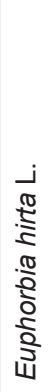 & 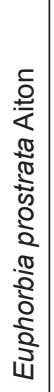 & 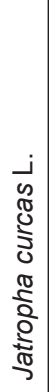 & 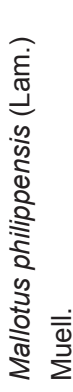 \\
\hline 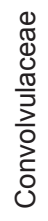 & $\begin{array}{l}0 \\
\mathbb{8} \\
\mathbb{8} \\
0 \\
\frac{\pi}{5} \\
\frac{1}{3} \\
30 \\
0 \\
0 \\
0 \\
0\end{array}$ & 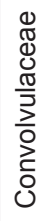 & 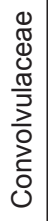 & 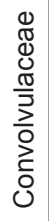 & 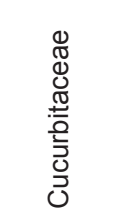 & 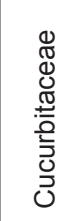 & 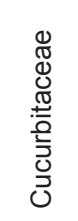 & 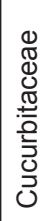 & 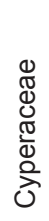 & 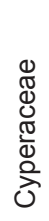 & 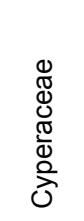 & 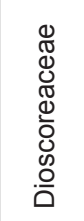 & 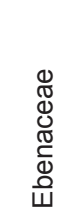 & 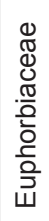 & 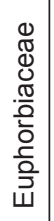 & 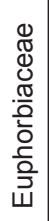 & 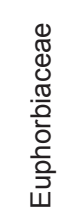 \\
\hline مْ & ம் & in & $\stackrel{\infty}{\circ}$ & ฉి & 8 & $\overline{6}$ & $\widetilde{0}$ & ஜृ & đீ & ஜூ & 8 & $\hat{0}$ & ${ }^{\infty}$ & 8 & 尺 & $\dot{\pi}$ & $\stackrel{N}{N}$ \\
\hline
\end{tabular}


Shrestha et al.: Flora of community managed forests

\begin{tabular}{|c|c|c|c|c|c|c|c|c|c|c|c|c|c|c|c|c|c|}
\hline 1 & ' & + & ' & 1 & + & I & + & ' & + & I & + & + & ' & I & ' & I & ' \\
\hline 1 & ' & + & + & ' & + & + & + & ' & + & 1 & + & + & I & + & + & I & + \\
\hline ' & I & + & + & ' & + & , & + & , & + & I & + & + & + & + & + & , & + \\
\hline+ & + & , & , & + & + & + & + & , & 1 & , & , & + & , & + & , & + & + \\
\hline+ & + & + & + & + & , & + & + & + & + & + & + & + & + & + & + & + & , \\
\hline$\omega$ & $\vdash$ & 0 & $I$ & $\vdash$ & I & $\vdash$ & $\infty$ & I & $I$ & $\vdash$ & $\omega$ & $\infty$ & $\infty$ & I & I & $\vdash$ & I \\
\hline $\mathbf{u}$ & U & 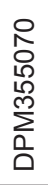 & 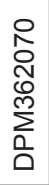 & $\mathrm{u}$ & $\begin{array}{l}\text { R } \\
\text { N } \\
\text { N } \\
\frac{0}{0} \\
0\end{array}$ & $\begin{array}{l}0 \\
0 \\
0 \\
0 \\
\sum_{0}^{m} \\
0\end{array}$ & 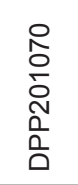 & U & $\begin{array}{l}0 \\
\stackrel{0}{ } \\
\stackrel{N}{N} \\
\sum_{0} \\
\Delta\end{array}$ & $\mathbf{v}$ & $\begin{array}{l}0 \\
0 \\
0 \\
\stackrel{0}{0} \\
\stackrel{1}{N} \\
\sum_{0} \\
0\end{array}$ & 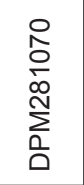 & 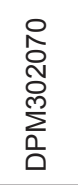 & 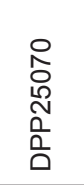 & 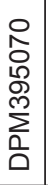 & $\mathbf{z}$ & $\begin{array}{l}0 \\
8 \\
\varnothing \\
0 \\
0 \\
0\end{array}$ \\
\hline $\begin{array}{l}\overline{\bar{z}} \\
\frac{\bar{T}}{\frac{\pi}{\alpha}} \\
\frac{\pi}{2}\end{array}$ & : $\frac{\text { 은 }}{\frac{1}{2}}$ & $\begin{array}{l}\overline{\bar{d}} \\
\bar{d} \\
\overline{\bar{\sigma}} \\
\bar{\nabla}\end{array}$ & & 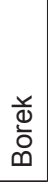 & $\begin{array}{l}\overline{\bar{z}} \\
\frac{1}{0} \\
\frac{0}{2} \\
\frac{1}{0} \\
\overline{0} \\
\sum\end{array}$ & $\begin{array}{l}\frac{0}{\pi} \\
. \overline{0} \\
\underline{\overline{0}}\end{array}$ & 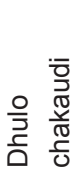 & & 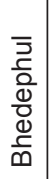 & 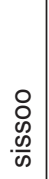 & 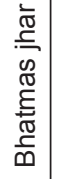 & 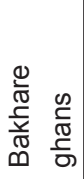 & 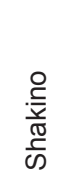 & 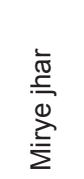 & & $\begin{array}{l}\frac{0}{0} \\
\frac{0}{\pi} \\
\frac{\bar{\sigma}}{\alpha}\end{array}$ & $\begin{array}{l}\stackrel{\Phi}{ \pm} \\
\frac{D}{ \pm} \\
\frac{D}{\square}\end{array}$ \\
\hline 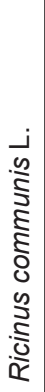 & 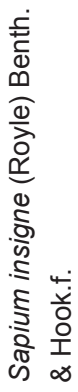 & 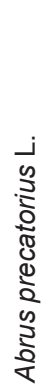 & 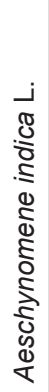 & 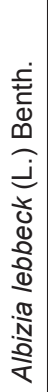 & 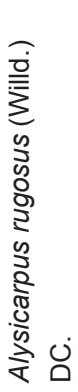 & 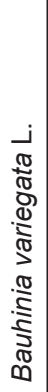 & 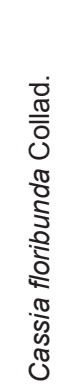 & 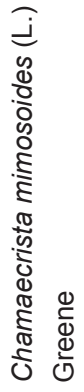 & $\begin{array}{l}\frac{5}{\overline{0}} \\
\frac{\alpha}{0} \\
\frac{\pi}{0} \\
\frac{0}{\pi} \\
\frac{\pi}{2} \\
\frac{\pi}{\pi} \\
\frac{\pi}{0} \\
0\end{array}$ & 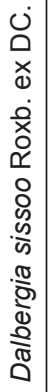 & 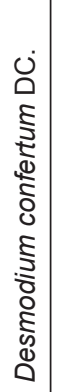 & 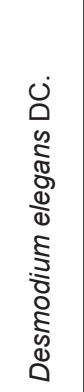 & 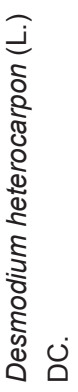 & 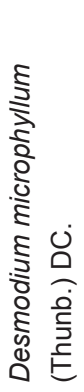 & 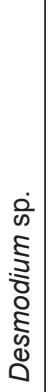 & 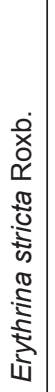 & 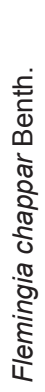 \\
\hline 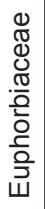 & 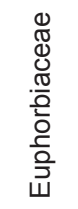 & 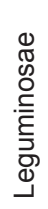 & 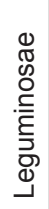 & 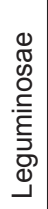 & 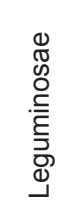 & 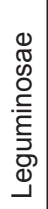 & 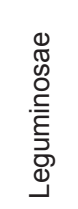 & 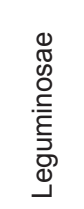 & 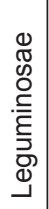 & 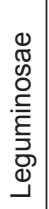 & 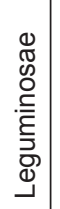 & 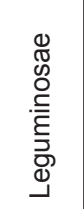 & 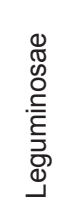 & 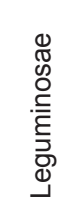 & 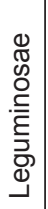 & 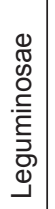 & 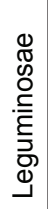 \\
\hline న & ポ & ம் & $\stackrel{0}{N}$ & $\hat{\imath}$ & $\stackrel{\infty}{\curvearrowright}$ & $R$ & ळ & $\infty$ & $\infty$ & $\infty$ & $\infty$ & $\infty$ & 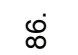 & ¿े & $\infty_{\infty}^{\infty}$ & $\infty$ & க் \\
\hline
\end{tabular}




\begin{tabular}{|c|c|c|c|c|c|c|c|c|c|c|c|c|c|c|c|c|c|c|}
\hline+ & + & + & I & + & 1 & + & 1 & 1 & + & 1 & + & + & + & 1 & + & + & I & + \\
\hline+ & + & + & + & + & + & + & ' & 1 & + & ' & + & + & + & + & + & + & + & + \\
\hline+ & + & + & + & + & + & + & + & ' & ' & ' & + & + & + & + & + & + & + & + \\
\hline+ & ' & + & 1 & 1 & 1 & + & ' & + & ' & + & + & + & + & ' & + & ' & ' & I \\
\hline+ & + & + & + & + & + & I & + & + & + & + & + & + & + & + & ' & + & + & + \\
\hline I & I & I & $\infty$ & 0 & $\infty$ & $\infty$ & $\vdash$ & $\vdash$ & $I$ & $\vdash$ & $\omega$ & $\omega$ & $\omega$ & I & $I$ & I & I & $I$ \\
\hline $\begin{array}{l}\text { 은 } \\
0 \\
0 \\
0 \\
0\end{array}$ & $\begin{array}{l}\text { ? } \\
\text { 은 } \\
\sum_{0}^{m} \\
0\end{array}$ & $\begin{array}{l}0 \\
0 \\
0 \\
\frac{m}{0} \\
0 \\
0\end{array}$ & $\begin{array}{l}\text { R } \\
0 \\
\text { م⿱ } \\
0 \\
\sum_{0}^{m} \\
0\end{array}$ & $\begin{array}{l}\text { P } \\
0 \\
0 \\
\text { D. } \\
\sum_{0}^{m} \\
0\end{array}$ & $\begin{array}{l}0 \\
0 \\
0 \\
\text { N } \\
\sum_{0} \\
0\end{array}$ & 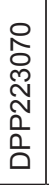 & 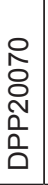 & U & $\begin{array}{l}\text { P } \\
\stackrel{N}{N} \\
\sum_{0}^{+} \\
0\end{array}$ & u & $\begin{array}{l}\text { ? } \\
0 \\
\text { in } \\
\sum_{0}^{m} \\
0\end{array}$ & $\begin{array}{l}0 \\
0 \\
0 \\
0 \\
\sum^{m} \\
\sum_{0} \\
0\end{array}$ & 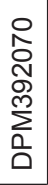 & $\begin{array}{l}0 \\
0 \\
i \\
\sum_{0}^{m} \\
\sum_{0}^{m} \\
0\end{array}$ & $\begin{array}{l}0 \\
0 \\
0 \\
0 \\
0 \\
0 \\
0 \\
0\end{array}$ & $\begin{array}{l}\text { ? } \\
0 \\
0 \\
\stackrel{N}{N} \\
\sum_{0} \\
0\end{array}$ & $\begin{array}{l}0 \\
0 \\
\circ \\
\infty \\
\infty \\
\\
0 \\
0\end{array}$ & 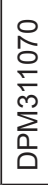 \\
\hline 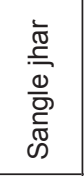 & & 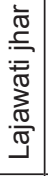 & $\begin{array}{l}\overline{\bar{\pi}} \\
\frac{\bar{\alpha}}{\bar{\alpha}}\end{array}$ & $\begin{array}{l}\frac{\Phi}{2} \\
\stackrel{D}{D} \\
\mathbb{D} \\
\infty\end{array}$ & $\begin{array}{l}\overline{\overline{0}} \\
\frac{\pi}{\pi} \\
\frac{2}{\pi} \\
\frac{2}{U}\end{array}$ & 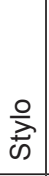 & $\overline{\overline{\bar{E}}}$ & $\begin{array}{l}\stackrel{\infty}{D} \\
\stackrel{n}{\pi}\end{array}$ & 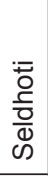 & 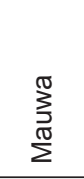 & 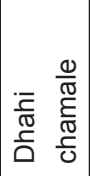 & $\begin{array}{l}\overline{\bar{z}} \\
\overline{\frac{T}{U}}\end{array}$ & 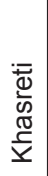 & 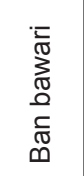 & $\begin{array}{l}\equiv \\
\Phi \\
\Phi \\
\stackrel{\infty}{5} \\
\stackrel{5}{5}\end{array}$ & 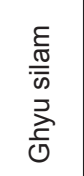 & 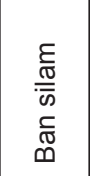 & $\begin{array}{l}\frac{0}{\overline{\frac{T}{2}}} \\
\frac{\partial}{\underline{\underline{\alpha}}}\end{array}$ \\
\hline 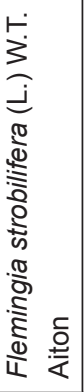 & 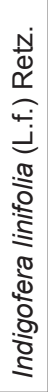 & 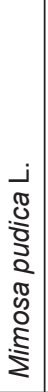 & 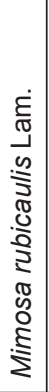 & 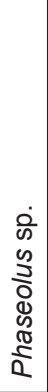 & 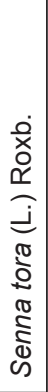 & 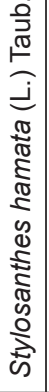 & 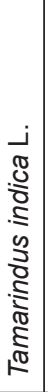 & 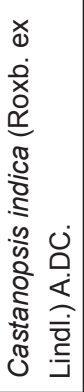 & $\begin{array}{l}\dot{5} \\
\frac{1}{0} \\
\mathbb{0} \\
0 \\
0 \\
0 \\
\frac{0}{0} \\
\frac{0}{0} \\
0 \\
0 \\
0 \\
0 \\
0 \\
0 \\
0 \\
0 \\
0 \\
0\end{array}$ & 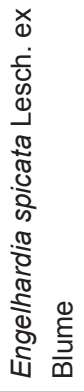 & 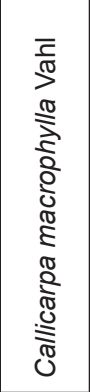 & 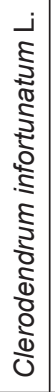 & 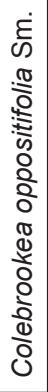 & 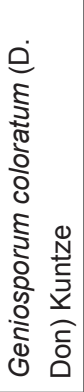 & 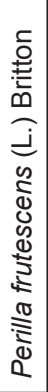 & 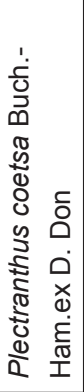 & 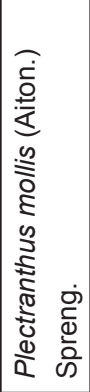 & 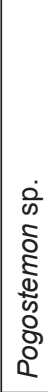 \\
\hline 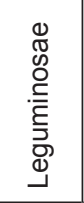 & 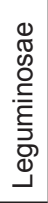 & 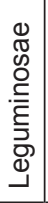 & 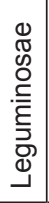 & 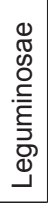 & 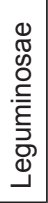 & 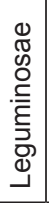 & 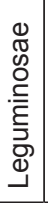 & 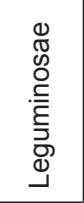 & 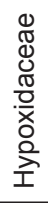 & 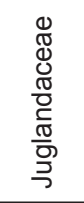 & 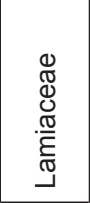 & 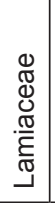 & 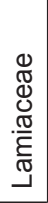 & 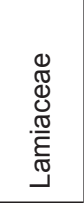 & 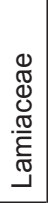 & 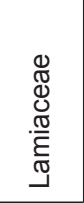 & 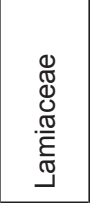 & 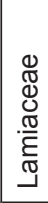 \\
\hline চ & ชิ & లু & ठా & ஜூ & $\mathscr{\varnothing}$ & ద & దீ & ৪ & 움 & 운 & ำ & 음 & & 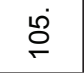 & 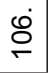 & $\stackrel{\circ}{\circ}$ & $\stackrel{\infty}{\circ}$ & 음 \\
\hline
\end{tabular}




\begin{tabular}{|c|c|c|c|c|c|c|c|c|c|c|c|c|c|c|c|c|c|}
\hline . & . & + & 1 & . & + & + & , & , & . & . &. & . & , & , & 1 & + & + \\
\hline , & 1 & + & ' & + & + & + & ' & ' & 1 & + & 1 & I & 1 & 1 & 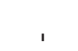 & + & + \\
\hline . & + & + & + & + & + & + & , & . & . & + & I & + & 1 & + & + & + & + \\
\hline , & ' & ' & ' & ' & , & + & + & + & , & + & . & , & , & , & + & + & + \\
\hline+ & + & + & + & + & + & + & + & + & + & ' & + & + & + & + & + & + & 1 \\
\hline$\vdash$ & I & I & I & I & I & $\infty$ & $\vdash$ & $\vdash$ & $I$ & I & $\omega$ & $\infty$ & $\vdash$ & $\omega$ & $\vdash$ & $\omega$ & $\omega$ \\
\hline U & 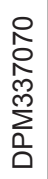 & 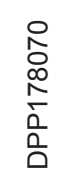 & $\begin{array}{l}\text { P } \\
\text { o } \\
\text { do } \\
\sum_{0}^{m} \\
0 \\
0\end{array}$ & 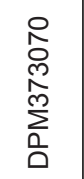 & 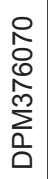 & 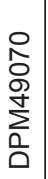 & U & $\mathcal{U}$ & U & $\begin{array}{l}\frac{0}{0} \\
0 \\
\frac{1}{2} \\
\frac{1}{0} \\
\frac{0}{0}\end{array}$ & U & 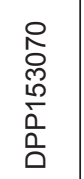 & U & 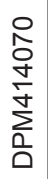 & $\begin{array}{l}0 \\
\text { O } \\
\text { 离 } \\
\sum_{0}^{0} \\
0\end{array}$ & $\begin{array}{l}R \\
0 \\
b \\
\dot{0} \\
\frac{0}{0} \\
0\end{array}$ & 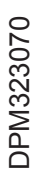 \\
\hline $\begin{array}{l}\text { 듬 } \\
\frac{0}{0} \\
\frac{0}{0}\end{array}$ & $\begin{array}{l}\stackrel{0}{\pi} \\
\stackrel{0}{\Xi} \\
\frac{0}{\sigma} \\
\stackrel{0}{0}\end{array}$ & $\begin{array}{l}\frac{0}{\pi} \\
\frac{2}{0} \\
\frac{0}{0} \\
\frac{0}{0}\end{array}$ & 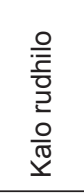 & & & 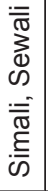 & 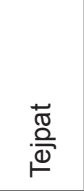 & 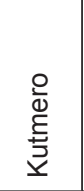 & 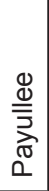 & 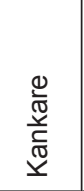 & 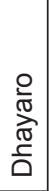 & 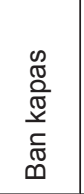 & $\frac{\overline{\widetilde{\sigma}}}{\bar{\omega}}$ & $\begin{array}{l}\mathbb{0} \\
\mathbb{0} \\
\stackrel{2}{\mathbb{N}} \\
\underline{\mathbb{T}}\end{array}$ & 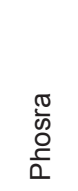 & 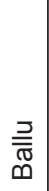 & 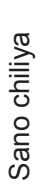 \\
\hline 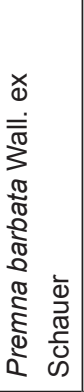 & 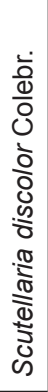 & 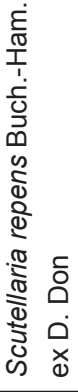 & 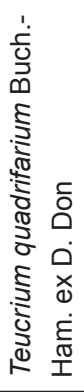 & 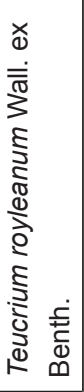 & 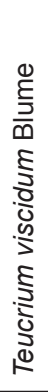 & 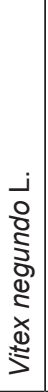 & 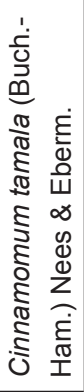 & 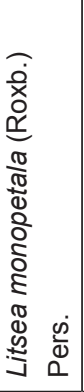 & 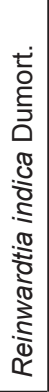 & 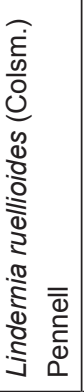 & 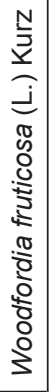 & 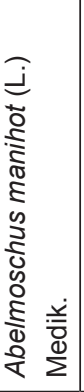 & 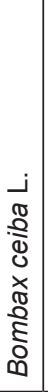 & 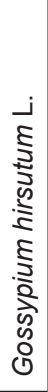 & 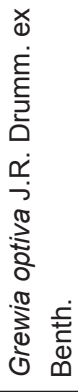 & 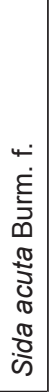 & 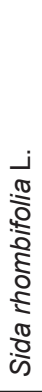 \\
\hline 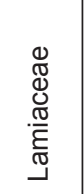 & 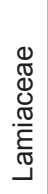 & 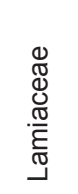 & 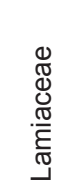 & 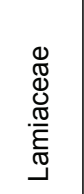 & 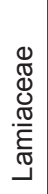 & 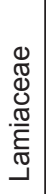 & 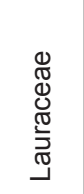 & 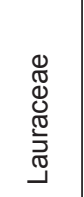 & 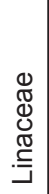 & 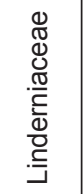 & 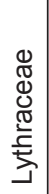 & 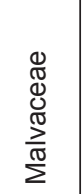 & 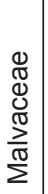 & 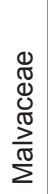 & 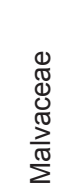 & 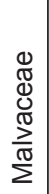 & 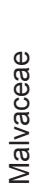 \\
\hline$\stackrel{\circ}{E}$ & $\dot{\check{E}}$ & $\stackrel{\mathfrak{N}}{\rightleftharpoons}$ & $\stackrel{\stackrel{m}{F}}{\rightleftharpoons}$ & $\stackrel{+}{\rightleftarrows}$ & $\stackrel{\text { மீ) }}{\rightleftharpoons}$ & $F$ & $\stackrel{\sim}{\Sigma}$ & $\stackrel{\infty}{\rightleftharpoons}$ & $\stackrel{\oplus}{\Gamma}$ & $\stackrel{\text { ํ் }}{\text {. }}$ & $\stackrel{\sim}{\simeq}$ & $\stackrel{\mathbb{N}}{\mathrm{N}}$ & $\stackrel{\mathscr{N}}{\leftarrow}$ & $\stackrel{\dot{\sim}}{\sim}$ & $\stackrel{\stackrel{\sim}{\sim}}{\sim}$ & $\stackrel{\stackrel{\oplus}{\sim}}{\sim}$ & $\stackrel{\grave{N}}{\sim}$ \\
\hline
\end{tabular}




\begin{tabular}{|c|c|c|c|c|c|c|c|c|c|c|c|c|c|c|c|c|c|c|c|c|}
\hline+ & + & + & + & + & + & ' & 1 & + & + & ' & ' & ' & ' & 1 & 1 & ' & 1 & ' & 1 & + \\
\hline+ & + & + & + & + & + & + & 1 & + & + & ' & ' & ' & ' & ' & + & ' & 1 & ' & I & + \\
\hline+ & + & + & + & + & + & + & ' & + & + & 1 & ' & ' & ' & ' & ' & 1 & 1 & 1 & 1 & + \\
\hline+ & + & + & + & ' & ' & + & + & + & + & ' & + & + & 1 & + & ' & + & + & + & + & + \\
\hline 1 & + & + & 1 & + & + & + & + & + & + & + & + & + & + & + & + & + & + & + & + & + \\
\hline I & $I$ & $\omega$ & $\omega$ & $\omega$ & $\infty$ & $\vdash$ & $\vdash$ & 0 & 0 & $\vdash$ & $\vdash$ & $\vdash$ & $\vdash$ & $\vdash$ & 0 & $\vdash$ & $\vdash$ & $\vdash$ & $\vdash$ & $\vdash$ \\
\hline 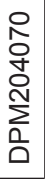 & 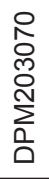 & 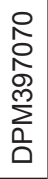 & 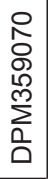 & $\begin{array}{l}\text { 은 } \\
\text { ᄋ } \\
\infty \\
\sum_{0}^{\infty} \\
0 \\
0\end{array}$ & $\begin{array}{l}\text { R } \\
\text { o } \\
\text { م) } \\
\sum_{0} \\
0\end{array}$ & 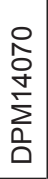 & $\mathbf{u}$ & 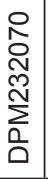 & 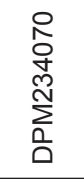 & U & z & $\mathbf{z}$ & $\mathrm{U}$ & $\mathbf{z}$ & $\begin{array}{l}\text { 응 } \\
\text { o } \\
0 \\
0 \\
0\end{array}$ & U & U & z & $\mathbf{u}$ & 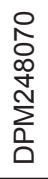 \\
\hline 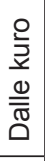 & 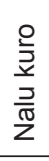 & 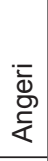 & $\begin{array}{l}\frac{\overline{0}}{\bar{D}} \\
\frac{1}{0} \\
0 \\
\frac{0}{0} \\
\infty \\
\end{array}$ & $\begin{array}{l}\frac{\bar{D}}{\overline{5}} \\
\frac{\bar{c}}{U} \\
\end{array}$ & 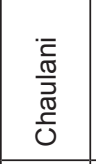 & 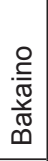 & $\overline{\bar{s}}$ & $\begin{array}{l}\frac{0}{ \pm} \\
\frac{0}{0} \\
\frac{0}{\pi} \\
\frac{\pi}{\pi} \\
\infty\end{array}$ & $\begin{array}{l}\text { 음 } \\
\overline{5} \\
\circ \\
\text { 음 } \\
\mathbb{1} \\
0\end{array}$ & $\begin{array}{l}\frac{1}{\pi} \\
\frac{\pi}{\pi} \\
\frac{\pi}{\pi} \\
\mathbb{D} \\
\infty\end{array}$ & $\begin{array}{l}\stackrel{\grave{\Phi}}{\bar{\oplus}} \\
\end{array}$ & $\begin{array}{l}\overline{5} \\
\frac{5}{\sqrt{0}} \\
0 \\
0\end{array}$ & 突 & $\begin{array}{l}\overline{\widetilde{\sigma}} \\
\stackrel{0}{0}\end{array}$ & $\begin{array}{l}\frac{0}{2} \\
\frac{2}{0} \\
\infty\end{array}$ & 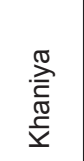 & $\begin{array}{l}\vec{\partial} \\
\underline{\underline{\underline{z}}} \\
\underline{\underline{y}}\end{array}$ & $\begin{array}{l}\frac{\pi}{\pi} \\
\mathscr{D} \\
\frac{\pi}{2}\end{array}$ & 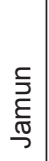 & $\begin{array}{l}\text { 독 } \\
\frac{\mathbf{v}}{\mathrm{J}}\end{array}$ \\
\hline 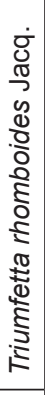 & 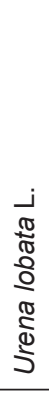 & 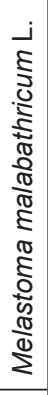 & 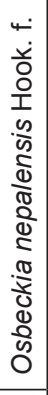 & 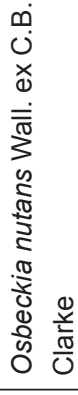 & 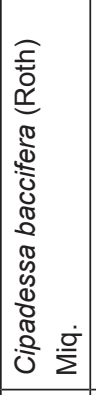 & 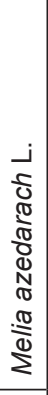 & 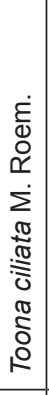 & 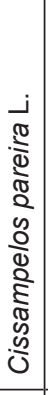 & 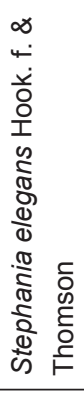 & 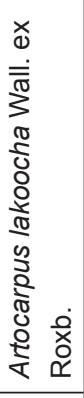 & 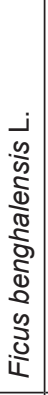 & 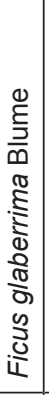 & 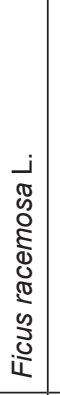 & 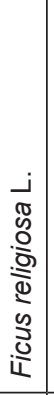 & 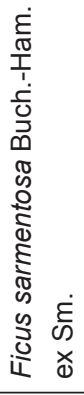 & 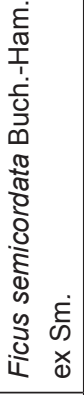 & 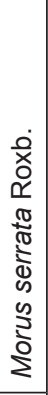 & 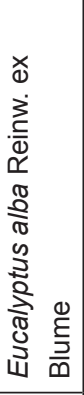 & 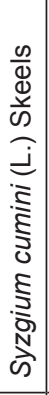 & 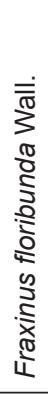 \\
\hline $\begin{array}{l}\mathbb{1} \\
\mathbb{N} \\
0 \\
0 \\
\frac{\pi}{2} \\
\frac{\pi}{\pi} \\
\sum\end{array}$ & 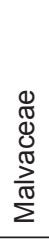 & 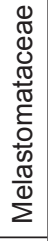 & 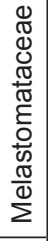 & 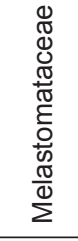 & 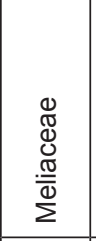 & 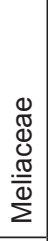 & 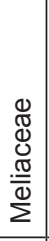 & 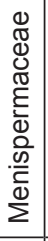 & 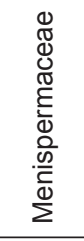 & 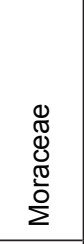 & $\begin{array}{l}\mathbb{\Xi} \\
\mathbb{\Xi} \\
0 \\
\mathbb{\pi} \\
\frac{0}{0} \\
\Sigma\end{array}$ & $\begin{array}{l}\mathbb{\Xi} \\
\mathbb{\pi} \\
0 \\
\mathbb{\pi} \\
\frac{0}{0} \\
\sum\end{array}$ & $\begin{array}{l}\mathbb{\Xi} \\
\mathbb{1} \\
\mathbb{0} \\
\mathbb{\pi} \\
\vdots 0 \\
\Sigma\end{array}$ & $\begin{array}{l}\mathbb{\Xi} \\
\mathbb{0} \\
\mathbb{0} \\
\widetilde{\pi} \\
\overline{0} \\
\Sigma\end{array}$ & $\begin{array}{l}\mathbb{0} \\
\mathbb{8} \\
0 \\
\mathbb{0} \\
\overline{0} \\
\Sigma\end{array}$ & $\begin{array}{l}0 \\
\mathbb{\pi} \\
0 \\
0 \\
\pi \\
\overline{0} \\
\Sigma\end{array}$ & 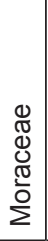 & 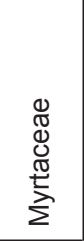 & 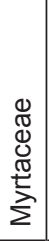 & 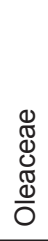 \\
\hline$\stackrel{\infty}{\stackrel{0}{\sim}}$ & $\stackrel{\stackrel{ }{ }}{\sim}$ & ஜ் & $\stackrel{\grave{m}}{\leftarrow}$ & $\stackrel{\text { }}{\sim}$ & $\stackrel{m}{=}$ & $\stackrel{\text { ले }}{\leftarrow}$ & $\stackrel{\text { ம் }}{\leftarrow}$ & $\begin{array}{l}\mathscr{m} \\
\stackrel{m}{\square}\end{array}$ & $\stackrel{\leftrightarrow}{\circ}$ & $\stackrel{\stackrel{\infty}{m}}{\stackrel{\infty}{\longrightarrow}}$ & $\stackrel{\text { ஜ் }}{\leftarrow}$ & $\begin{array}{l}\dot{⿱} \\
+\end{array}$ & $\stackrel{\dot{v}}{\square}$ & $\underset{\stackrel{\mathcal{J}}{\leftarrow}}{ }$ & $\stackrel{\dot{g}}{\sim}$ & $\underset{\forall}{\stackrel{J}{\leftarrow}}$ & 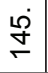 & $\stackrel{\dot{\theta}}{\leftarrow}$ & 守 & 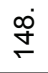 \\
\hline
\end{tabular}


Shrestha et al.: Flora of community managed forests

\begin{tabular}{|c|c|c|c|c|c|c|c|c|c|c|c|c|c|c|c|c|c|c|}
\hline ' & + & + & ' & + & ' & I & + & + & I & ' & 1 & I & + & ' & ' & . & + & ' \\
\hline ' & + & + & ' & + & I & I & + & + & I & 1 & 1 & 1 & + & + & ' & . & + & ' \\
\hline+ & + & + & 1 & + & , & , & + & + & + & + &, & 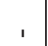 & + & + & + &. & + & ' \\
\hline+ & . & + & + & + & + & + & ' & + & + & + & 1 & + & + & + & + & + & + & ' \\
\hline+ & + & + & + & + & + & + & + & + & 1 & + & + & + & 1 & ' & ' & + & + & + \\
\hline I & $\omega$ & $\vdash$ & $\vdash$ & 0 & I & $\vdash$ & 0 & I & I & $I$ & $I$ & I & I & I & I & $I$ & I & I \\
\hline 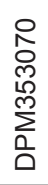 & 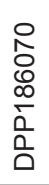 & $\begin{array}{l}0 \\
0 \\
8 \\
\varnothing 0 \\
0 \\
0 \\
0\end{array}$ & $\mathbf{z}$ & $\begin{array}{l}0 \\
0 \\
o \\
\infty \\
\infty \\
\sum_{0}^{m} \\
\Delta\end{array}$ & $\mathbf{u}$ & $\mathbf{z}$ & $\begin{array}{l}0 \\
0 \\
0 \\
0 \\
\sum_{0}^{m} \\
0\end{array}$ & $\begin{array}{l}\frac{0}{0} \\
\frac{+}{+} \\
\frac{1}{n} \\
\frac{0}{0}\end{array}$ & $\begin{array}{l}\frac{0}{0} \\
\frac{0}{\infty} \\
0 \\
0 \\
0\end{array}$ & $\begin{array}{l}0 \\
\hat{0} \\
\hat{N} \\
\text { N } \\
\text { D } \\
0\end{array}$ & $\mathbf{z}$ & $\mathbf{u}$ & 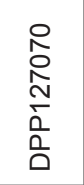 & $\begin{array}{l}\text { D } \\
\text { م } \\
\text { N̂} \\
\sum_{0}^{m} \\
0\end{array}$ & 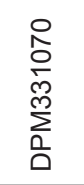 & U & $\begin{array}{l}0 \\
\frac{0}{0} \\
\frac{0}{0} \\
\frac{0}{0} \\
\frac{1}{0}\end{array}$ & U \\
\hline $\begin{array}{l}\frac{0}{\overline{\frac{E}{\sigma}}} \\
\frac{\sigma}{\bar{\sigma}} \\
\frac{\bar{\sigma}}{U} \\
\end{array}$ & 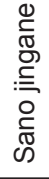 & 衮 & $\begin{array}{l}\frac{\pi}{\pi} \\
\frac{\varepsilon}{<}\end{array}$ & 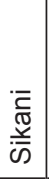 & 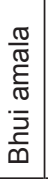 & $\begin{array}{l}\frac{\mathscr{D}}{\overline{\widetilde{N}}} \\
\stackrel{2}{1}\end{array}$ & $\frac{\pi}{\frac{0}{2}}$ & 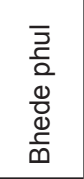 & $\begin{array}{l}\bar{D} \\
\frac{\delta}{\sigma} \\
\underline{\sigma} \\
\underline{\underline{\sigma}}\end{array}$ & & $\begin{array}{l}\frac{\pi}{\omega} \\
\frac{\pi}{\pi} \\
\underline{\pi}\end{array}$ & $\begin{array}{l}\text { D } \\
\stackrel{D}{\mathbb{N}} \\
\infty\end{array}$ & & & & $\stackrel{\circ}{\circ}$ & & $\begin{array}{l}\stackrel{\infty}{\mathbb{N}} \\
\stackrel{\infty}{\infty}\end{array}$ \\
\hline 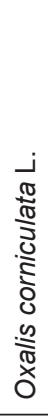 & 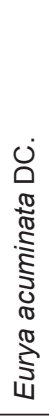 & 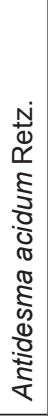 & 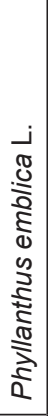 & 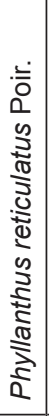 & 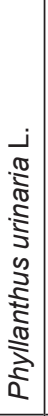 & 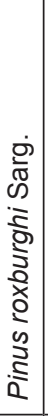 & $\begin{array}{l}\dot{j} \\
5 \\
\mathbf{2} \\
\vdots \\
\frac{0}{1} \\
\Phi \\
\frac{2}{2}\end{array}$ & 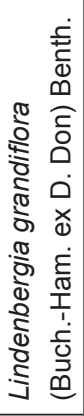 & 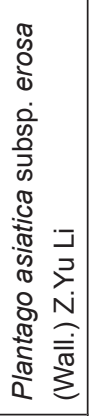 & 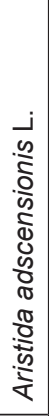 & 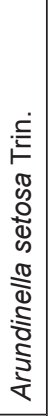 & 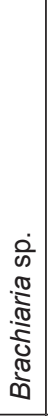 & 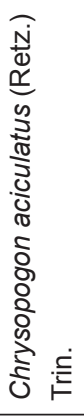 & 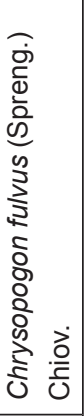 & 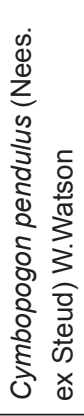 & 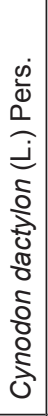 & 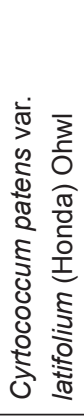 & 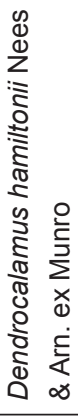 \\
\hline 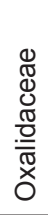 & 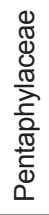 & 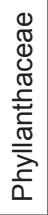 & 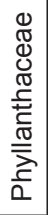 & 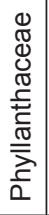 & 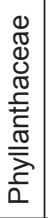 & 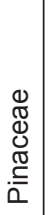 & 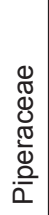 & 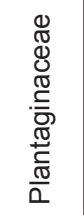 & 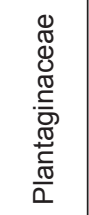 & $\begin{array}{l}\mathbb{1} \\
\mathbb{E} \\
0 \\
\mathbb{\Xi} \\
0\end{array}$ & $\begin{array}{l}0 \\
\mathbb{J} \\
\mathbb{J} \\
\mathbb{D} \\
0 \\
0\end{array}$ & $\begin{array}{l}\mathbb{\Xi} \\
\mathbb{\Xi} \\
\mathscr{\Xi} \\
\mathbb{\Xi} \\
0\end{array}$ & $\begin{array}{l}\mathbb{1} \\
\mathbb{8} \\
\mathbb{J} \\
\mathbb{D} \\
0\end{array}$ & $\begin{array}{l}\mathbb{\Xi} \\
\mathbb{\Xi} \\
\mathbb{\Xi} \\
\mathbb{D} \\
0\end{array}$ & $\begin{array}{l}\mathbb{D} \\
\mathbb{0} \\
0 \\
\mathbb{0} \\
0\end{array}$ & $\begin{array}{l}\mathbb{D} \\
\mathbb{N} \\
0 \\
\mathbb{D} \\
0 \\
0\end{array}$ & $\begin{array}{l}\mathbb{1} \\
\mathbb{N} \\
0 \\
\mathbb{0} \\
0 \\
\square\end{array}$ & $\begin{array}{l}\mathbb{\Xi} \\
\mathbb{\Xi} \\
\mathbb{\Xi} \\
\mathbb{D} \\
0\end{array}$ \\
\hline$\underset{⿱ 亠 巾}{+}$ & ட் & $\stackrel{5}{\circ}$ & กิ & ֻొ & 点 & 占 & $\stackrel{60}{\sim}$ & $\stackrel{5}{5}$ & $\stackrel{12}{\square}$ & $\stackrel{60}{\sim}$ & $\ddot{8}$ & $\stackrel{\varphi}{\sim}$ & ֻู & రृ & 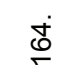 & $\stackrel{0}{0}$ & $\ddot{\theta}$ & 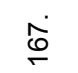 \\
\hline
\end{tabular}




\begin{tabular}{|c|c|c|c|c|c|c|c|c|c|c|c|c|c|c|c|}
\hline ' & I & ' & + & + & + & ' & + & ' & I & + & + & + & + & I & ' \\
\hline I & ' & ' & + & + & + & ' & + & 1 & + & + & + & + & + & + & , \\
\hline+ & , & + & + & + & + & + & + & , & + & + & + & + & + & + & + \\
\hline ' & + & 1 & + & + & + & + & + & + & + & + & + & + & + & + & + \\
\hline+ & + & + & ' & + & 1 & + & + & + & + & + & + & + & + & + & + \\
\hline$I$ & I & $I$ & $I$ & I & I & I & I & I & $I$ & I & $\infty$ & 0 & $I$ & $\vdash$ & I \\
\hline 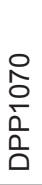 & U & 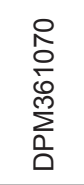 & 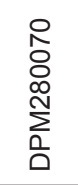 & $\begin{array}{l}\text { 尺 } \\
\text { م } \\
\text { N్} \\
\sum_{0}^{0} \\
0\end{array}$ & $\begin{array}{l}0 \\
0 \\
o \\
0 \\
\stackrel{N}{N} \\
\sum_{0} \\
\Delta\end{array}$ & 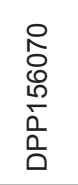 & 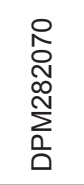 & u & $\begin{array}{l}\text { R } \\
\text { o } \\
\stackrel{0}{N} \\
\stackrel{\Lambda}{n} \\
\stackrel{0}{0}\end{array}$ & $\begin{array}{l}\text { R } \\
\text { o } \\
\text { m } \\
\text { n. } \\
\text { م. }\end{array}$ & 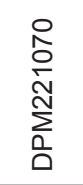 & $\begin{array}{l}0 \\
0 \\
0 \\
0 \\
\sum_{0}^{m} \\
\Delta\end{array}$ & $\begin{array}{l}0 \\
\frac{0}{0} \\
\frac{1}{n} \\
\frac{1}{0} \\
0\end{array}$ & 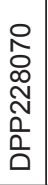 & $\begin{array}{l}\circ \\
0 \\
0 \\
0 \\
0 \\
0 \\
0 \\
0\end{array}$ \\
\hline & $\stackrel{2}{n}$ & & & 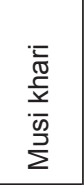 & & & & 另 & $\stackrel{\varrho}{\stackrel{2}{L}}$ & 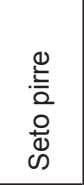 & $\begin{array}{l}\overline{\bar{c}} \\
\frac{\bar{\sigma}}{\omega} \\
\overline{\bar{m}}\end{array}$ & 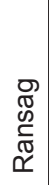 & 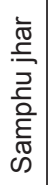 & 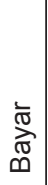 & 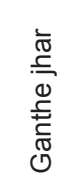 \\
\hline 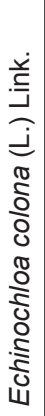 & 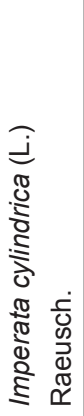 & 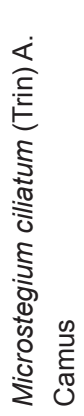 & 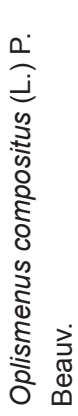 & 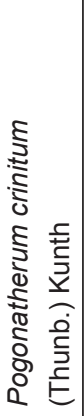 & 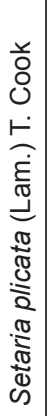 & 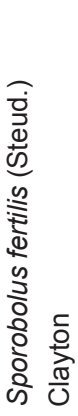 & 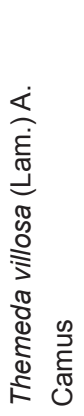 & 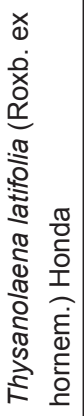 & 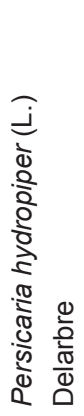 & 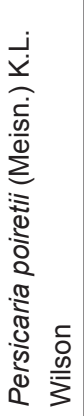 & 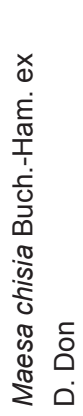 & 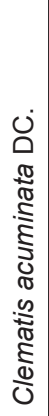 & 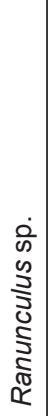 & 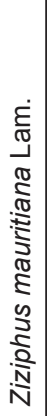 & 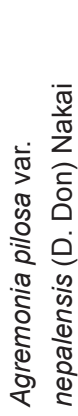 \\
\hline $\begin{array}{l}0 \\
\mathbb{D} \\
\mathbb{J} \\
0 \\
\mathbb{Z} \\
0 \\
0\end{array}$ & $\begin{array}{l}\mathbb{0} \\
\mathbb{\pi} \\
\mathbb{0} \\
\mathbb{\pi} \\
0 \\
0\end{array}$ & $\begin{array}{l}\mathbb{0} \\
\mathbb{\pi} \\
\mathbb{1} \\
\mathbb{\pi} \\
0 \\
0\end{array}$ & $\begin{array}{l}\mathbb{0} \\
\mathbb{\pi} \\
\mathbb{1} \\
\mathbb{0} \\
0 \\
0\end{array}$ & 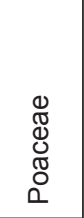 & 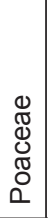 & $\begin{array}{l}\mathbb{0} \\
\mathbb{\pi} \\
\mathbb{1} \\
\mathbb{\Xi} \\
0 \\
0\end{array}$ & 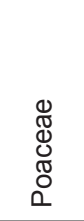 & 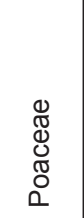 & 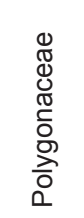 & $\begin{array}{l}\mathbb{0} \\
\mathbb{8} \\
0 \\
\mathbb{0} \\
\check{0} \\
\stackrel{0}{0} \\
0\end{array}$ & 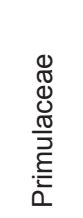 & 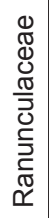 & 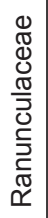 & 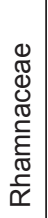 & 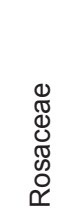 \\
\hline $\begin{array}{l}0 \\
\stackrel{0}{0}\end{array}$ & ஜ் & $\stackrel{\circ}{\stackrel{2}{\Sigma}}$ & $\stackrel{\dot{\Sigma}}{\Sigma}$ & $\stackrel{\mathfrak{N}}{\stackrel{2}{\sigma}}$ & $\stackrel{\stackrel{N}{N}}{\Sigma}$ & $\stackrel{+}{\stackrel{+}{\sigma}}$ & $\stackrel{\stackrel{\rho}{\circ}}{\stackrel{2}{\sigma}}$ & $\stackrel{\oplus}{\stackrel{0}{\sim}}$ & $\stackrel{\hat{N}}{\sigma}$ & $\stackrel{\infty}{\stackrel{\infty}{\sim}}$ & $\stackrel{\circ}{\stackrel{2}{\sim}}$ & $\stackrel{\infty}{\infty}$ & $\stackrel{\dot{\infty}}{\sigma}$ & $\stackrel{\infty}{\sim}$ & $\stackrel{\infty}{\infty}$ \\
\hline
\end{tabular}




\begin{tabular}{|c|c|c|c|c|c|c|c|c|c|c|c|c|c|c|c|c|c|c|}
\hline ' & ' & 1 & 1 & 1 & ' & + & ' & . & ' & ' & ' & ' & ' & 1 & + & ' & I & + \\
\hline+ & ' & + & + & + & + & + & ' & + & ' & ' & ' & ' & 1 & + & + & + & ' & + \\
\hline+ & 1 & + & 1 & + & + & + & ' & 1 & ' & . & ' & ' & ' & + & + & + & + & + \\
\hline+ & + & ' & + & + & + & ' & ' & + & ' & + & + & + & ' & + & ' & I & + & + \\
\hline ' & + & + & + & + & ' & + & + & + & + & + & + & + & + & + & + & + & + & I \\
\hline I & $\vdash$ & $\omega$ & 0 & I & I & I & $\infty$ & $\vdash$ & $\omega$ & $\vdash$ & $\vdash$ & 0 & $I$ & $I$ & $\vdash$ & $I$ & I & $\omega$ \\
\hline 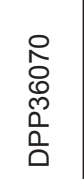 & U & 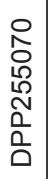 & 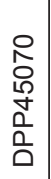 & $\begin{array}{l}0 \\
0 \\
0 \\
6 \\
0 \\
0 \\
0\end{array}$ & $\begin{array}{l}0 \\
\text { o } \\
\sum_{0}^{m} \\
0 \\
0\end{array}$ & $\begin{array}{l}0 \\
0 \\
0 \\
\stackrel{D}{N} \\
\sum_{0} \\
0\end{array}$ & $\mathbf{u}$ & $\begin{array}{l}0 \\
0 \\
0 \\
\text { O } \\
\sum_{0} \\
0 \\
0\end{array}$ & U & $\mathbf{u}$ & U & U & $\mathbf{0}$ & $\begin{array}{l}0 \\
0 \\
0 \\
m \\
0 \\
0 \\
0\end{array}$ & 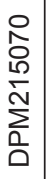 & $\begin{array}{l}0 \\
0 \\
0 \\
\stackrel{d}{N} \\
\sum_{0} \\
0\end{array}$ & $\begin{array}{l}\text { R } \\
\text { O } \\
\stackrel{N}{N} \\
\stackrel{1}{0} \\
0\end{array}$ & 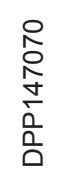 \\
\hline 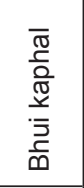 & $\begin{array}{l}\bar{\pi} \\
\stackrel{\pi}{\pi} \\
\sum\end{array}$ & 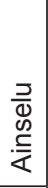 & 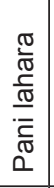 & $\begin{array}{l}: \overline{\overline{0}} \\
\overline{0} \\
\text { 듬 }\end{array}$ & $\begin{array}{l}\frac{1}{\pi} \\
\stackrel{5}{0} \\
\frac{0}{\pi} \\
\frac{2}{2} \\
\frac{2}{0} \\
\frac{5}{0} \\
\end{array}$ & 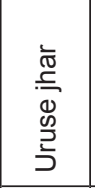 & 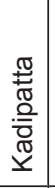 & $\begin{array}{l}\frac{\bar{x}}{\bar{z}} \\
\overline{0} \\
\frac{5}{5} \\
z\end{array}$ & $\begin{array}{l}\bar{\vdots} \\
\bar{c} \\
\overline{0} \\
\mathbb{0} \\
\text { I }\end{array}$ & 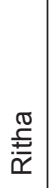 & 彦 & 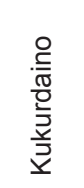 & 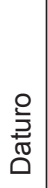 & 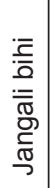 & $\begin{array}{l}\frac{\pi}{\sigma} \\
\frac{\sigma}{0} \\
0\end{array}$ & 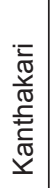 & 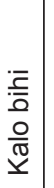 & \\
\hline 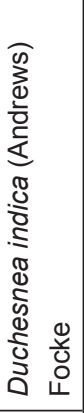 & 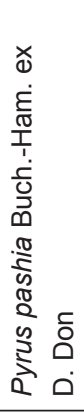 & 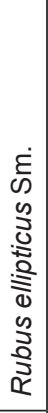 & 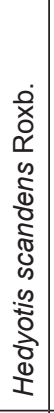 & 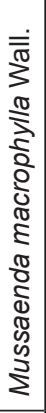 & 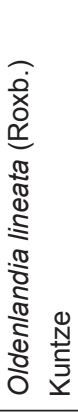 & 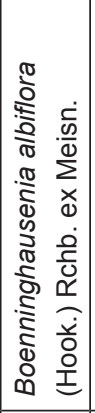 & 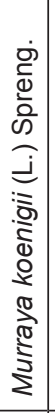 & 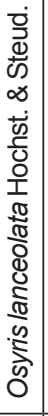 & 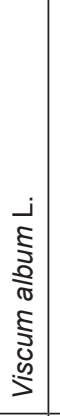 & 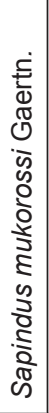 & 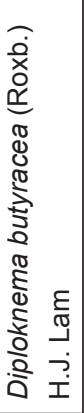 & 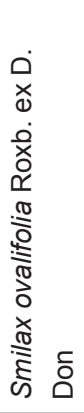 & 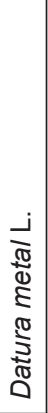 & 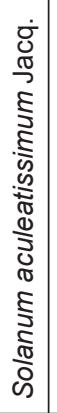 & 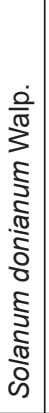 & 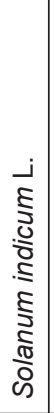 & 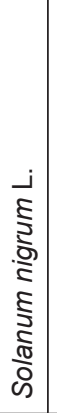 & 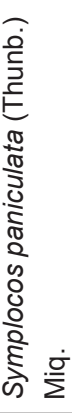 \\
\hline 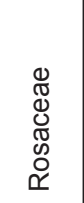 & 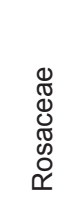 & 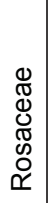 & 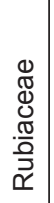 & 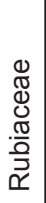 & 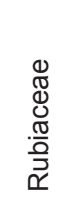 & 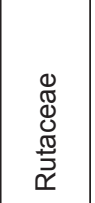 & 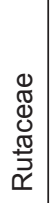 & 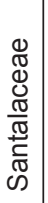 & 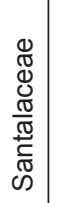 & 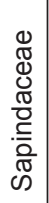 & 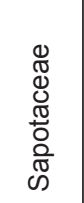 & 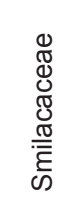 & $\begin{array}{l}\mathbb{D} \\
\mathbb{J} \\
0 \\
\mathbb{\pi} \\
\frac{\pi}{\pi} \\
\frac{\pi}{0} \\
\dot{\infty}\end{array}$ & $\begin{array}{l}\mathbb{\Xi} \\
\mathbb{\pi} \\
\mathbb{0} \\
\mathbb{\pi} \\
\frac{\pi}{\pi} \\
\frac{0}{0} \\
\mathcal{\infty}\end{array}$ & 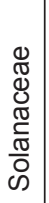 & 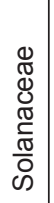 & 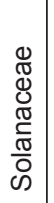 & 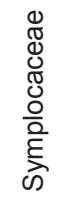 \\
\hline$\stackrel{+}{\infty}$ & $\stackrel{\stackrel{\circ}{0}}{\infty}$ & $\begin{array}{l}\mathscr{\infty} \\
\infty\end{array}$ & 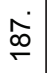 & $\stackrel{\infty}{\infty}$ & ஹ & த் & $\stackrel{\check{\sigma}}{\square}$ & ๙઼่ & $\stackrel{\text { ஜ் }}{\circ}$ & $\stackrel{Ð}{\circ}$ & 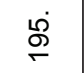 & $\stackrel{\mathscr{8}}{-}$ & $\stackrel{\text { Ф̊ }}{\square}$ & $\stackrel{\circ}{\circ}$ & $\stackrel{\text { g }}{\circ}$ & ठ் & $\bar{\sim}$ & ণั่ \\
\hline
\end{tabular}




\begin{tabular}{|c|c|c|c|c|c|c|c|c|c|c|c|}
\hline+ & + & + & + & + & ' & + & ' & 1 & ' & ' & $\infty$ \\
\hline+ & + & + & + & + & + & + & 1 & + & , & + & $\stackrel{\stackrel{\sim}{\sim}}{ }$ \\
\hline+ & + & + & + & + & + & + & + & 1 & , & + & 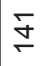 \\
\hline+ & ' & + & ' & + & ' & + & + & + & + & , & $\stackrel{?}{\sim}$ \\
\hline+ & + & + & + & I & + & , & , & + & + & + & $\stackrel{0}{\stackrel{0}{\sim}}$ \\
\hline$\vdash$ & $\omega$ & $\omega$ & I & I & I & 0 & $\omega$ & 0 & $I$ & I & \\
\hline 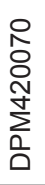 & 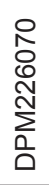 & 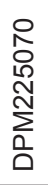 & 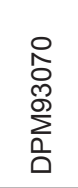 & 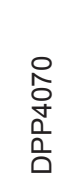 & 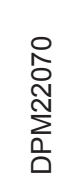 & $\begin{array}{l}\text { 을 } \\
0 \\
\llcorner 0 \\
0 \\
0 \\
0 \\
0\end{array}$ & $\begin{array}{l}\text { D } \\
0 \\
0 \\
\text { Nิ } \\
\sum_{0}^{0} \\
0\end{array}$ & $\begin{array}{l}\text { D } \\
0 \\
\infty \\
\stackrel{2}{1} \\
\sum_{0} \\
0\end{array}$ & U & $\begin{array}{l}0 \\
\text { O } \\
\text { O } \\
\sum_{0} \\
0 \\
0\end{array}$ & \\
\hline $\begin{array}{l}\stackrel{0}{5} \\
\frac{\pi}{5} \\
\frac{\pi}{\frac{\pi}{J}} \\
\end{array}$ & 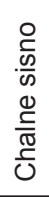 & $\begin{array}{l}\frac{0}{E} \\
\frac{\mathbb{N}}{\check{1}}\end{array}$ & 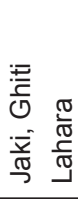 & $\begin{array}{l}\frac{\pi}{\frac{\pi}{\pi}} \\
\frac{\frac{\pi}{\pi}}{\sigma} \\
\frac{\pi}{\pi} \\
0\end{array}$ & $\frac{\frac{0}{2}}{\frac{O}{U}}$ & 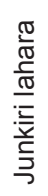 & $\begin{array}{l}\overline{\bar{\sigma}} \\
\frac{\bar{\omega}}{\pi} \\
\overline{0}\end{array}$ & 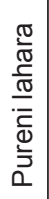 & 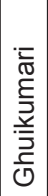 & 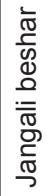 & \\
\hline 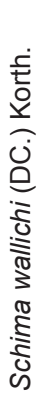 & 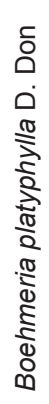 & 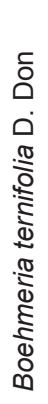 & 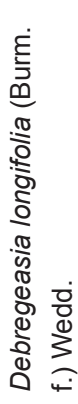 & 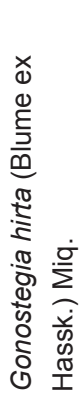 & 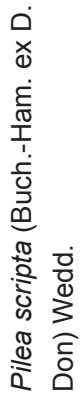 & 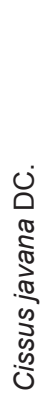 & 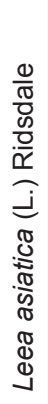 & 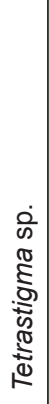 & 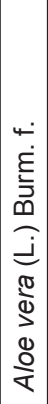 & 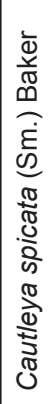 & \\
\hline $\begin{array}{l}\mathbb{1} \\
\mathbb{D} \\
\mathbb{0} \\
\mathbb{J} \\
\mathbb{1} \\
\stackrel{F}{F}\end{array}$ & 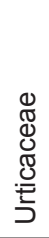 & 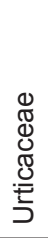 & 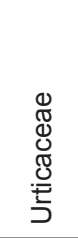 & 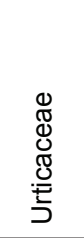 & 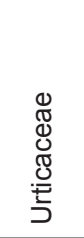 & 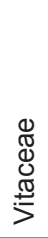 & 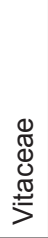 & $\begin{array}{l}\mathbb{0} \\
\mathbb{N} \\
0 \\
\mathbb{J} \\
\stackrel{\Xi}{>}\end{array}$ & 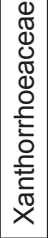 & 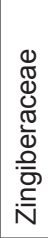 & $\begin{array}{l}\bar{\pi} \\
\stackrel{\overline{0}}{\circ}\end{array}$ \\
\hline$\curvearrowright$ & ठ্ণ & ஜ̊ & ஜें & $\hat{\sim}$ & $\stackrel{\infty}{\circ}$ & ஓं & $\stackrel{\dot{N}}{\stackrel{0}{ }}$ & $\dot{\sim}$ & $\stackrel{\stackrel{N}{N}}{\sim}$ & $\frac{\dot{m}}{\sim}$ & \\
\hline
\end{tabular}

